\title{
Binding affinity landscapes constrain the evolution of broadly neutralizing anti- influenza antibodies
}

\author{
Angela M Phillips ${ }^{1 \dagger}$, Katherine R Lawrence ${ }^{1,2,3,4 \dagger}$, Alief Moulana ${ }^{1}$, Thomas Dupic ${ }^{1}$, \\ Jeffrey Chang ${ }^{5}$, Milo S Johnson ${ }^{1}$, Ivana Cvijovic ${ }^{6}$, Thierry Mora ${ }^{7}$, \\ Aleksandra M Walczak ${ }^{7}$, Michael M Desai ${ }^{1,2,3,5 *}$
}

${ }^{1}$ Department of Organismic and Evolutionary Biology, Harvard University, Cambridge, United States; ${ }^{2}$ NSF-Simons Center for Mathematical and Statistical Analysis of Biology, Harvard University, Cambridge, United States; ${ }^{3}$ Quantitative Biology Initiative, Harvard University, Cambridge, United States; ${ }^{4}$ Department of Physics, Massachusetts Institute of Technology, Cambridge, United States; ${ }^{5}$ Department of Physics, Harvard University, Cambridge, United States; ${ }^{6}$ Department of Applied Physics, Stanford University, Stanford, United States; ${ }^{7}$ Laboratoire de physique de ÍÉcole Normale Supérieure, CNRS, PSL University, Sorbonne Université, and Université de Paris, Paris, France

*For correspondence: mdesai@oeb.harvard.edu

${ }^{\dagger}$ These authors contributed equally to this work

Competing interest: See page 25

Funding: See page 25

Received: 17 June 2021

Preprinted: 25 May 2021

Accepted: 05 September 2021

Published: 07 September 2021

Reviewing editor: Sarel Jacob Fleishman, Weizmann Institute of Science, Israel

(c) Copyright Phillips et al. This article is distributed under the terms of the Creative Commons Attribution License, which permits unrestricted use and redistribution provided that the original author and source are credited.

\begin{abstract}
Over the past two decades, several broadly neutralizing antibodies (bnAbs) that confer protection against diverse influenza strains have been isolated. Structural and biochemical characterization of these bnAbs has provided molecular insight into how they bind distinct antigens. However, our understanding of the evolutionary pathways leading to bnAbs, and thus how best to elicit them, remains limited. Here, we measure equilibrium dissociation constants of combinatorially complete mutational libraries for two naturally isolated influenza bnAbs (CR9114, 16 heavy-chain mutations; CR6261, 11 heavy-chain mutations), reconstructing all possible evolutionary intermediates back to the unmutated germline sequences. We find that these two libraries exhibit strikingly different patterns of breadth: while many variants of CR6261 display moderate affinity to diverse antigens, those of CR9114 display appreciable affinity only in specific, nested combinations. By examining the extensive pairwise and higher order epistasis between mutations, we find key sites with strong synergistic interactions that are highly similar across antigens for CR6261 and different for CR9114. Together, these features of the binding affinity landscapes strongly favor sequential acquisition of affinity to diverse antigens for CR9114, while the acquisition of breadth to more similar antigens for CR6261 is less constrained. These results, if generalizable to other bnAbs, may explain the molecular basis for the widespread observation that sequential exposure favors greater breadth, and such mechanistic insight will be essential for predicting and eliciting broadly protective immune responses.
\end{abstract}

\section{Introduction}

Vaccination harnesses the adaptive immune system, which responds to new pathogens by mutating antibody-encoding genes and selecting for variants that bind the pathogen of interest. However, influenza remains a challenging target for immunization: most antibodies elicited by vaccines provide protection against only a subset of strains, largely due to the rapid evolution of the influenza surface protein hemagglutinin (HA) (Wiley et al., 1981; Smith et al., 2004). After nearly two decades of studies, numerous broadly neutralizing antibodies (bnAbs) have been isolated from humans, with 
varying degrees of cross-protection against diverse strains (Corti et al., 2017; Throsby et al., 2008; Dreyfus et al., 2012; Corti et al., 2011; Schmidt et al., 2015). Still, we do not fully understand many factors affecting how and when bnAbs are produced. In particular, affinity is acquired through a complex process of mutation and selection (Victora and Nussenzweig, 2012), but the effects of mutations on binding affinity to diverse antigens are not well characterized.

For example, consider two well-studied influenza bnAbs that display varying levels of breadth: CR9114 is one of the broadest anti-influenza antibodies ever found, neutralizing strains from both groups of influenza A and strains from influenza B, while CR6261 is limited to neutralizing strains from Group 1 of influenza A (Throsby et al., 2008; Dreyfus et al., 2012; Ekiert et al., 2009; Lingwood et al., 2012). Both antibodies were isolated from vaccinated donors, derive from very similar germline sequences (IGHV1 -69 and IGHJ6), and bind the conserved HA stem epitope (Figure 1-figure supplement 3; Throsby et al., 2008; Dreyfus et al., 2012; Ekiert et al., 2009). Each antibody heavy chain has many mutations (18 amino acid changes for CR9114, 14 for CR6261, Figure 1A), including seven positions that are mutated in both, yet the contributions of these mutations to affinity against different antigens remain unclear (Dreyfus et al., 2012; Avnir et al., 2014).

Beyond single mutational effects, it remains unknown whether there are correlated effects or strong trade-offs between binding to different antigens (pleiotropy), or non-additive interactions between mutations (epistasis). Such epistatic and pleiotropic effects can constrain the mutational pathways accessible under selection, as has been observed for other proteins (Weinreich et al., 2006; Starr et al., 2017; Ortlund et al., 2007; Podgornaia and Laub, 2015; Gong et al., 2013; Sailer and Harms, 2017b; Miton and Tokuriki, 2016; Poelwijk et al., 2019; Bank et al., 2015). Epistasis in antibody-antigen interactions remains significantly understudied (Adams et al., 2019; Pappas et al., 2014; Braden et al., 1998) and most deep mutational scanning studies have focused on antigens (Doud et al., 2018; Wu et al., 2020; Starr et al., 2021). In contrast to typical protein evolution, antibody affinity maturation proceeds by discrete rounds of mutation and selection (Victora and Nussenzweig, 2012), typically with more than one nucleotide mutation occurring between selective rounds (Unniraman and Schatz, 2007). In addition, antibodies are inherently mutationally tolerant (Braden et alı, 1998; Chen et al., 1999; Burks et al., 1997; Corti and Lanzavecchia, 2013; Klein et al., 2013), generating opportunities for interactions that scale combinatorially. Thus, if epistatic and pleiotropic constraints exist for antibodies, they could affect the likelihood of producing bnAbs under different antigen selection regimes (Pappas et al., 2014) and may account for the low frequencies of bnAbs in natural repertoires (Corti et al., 2017). Characterizing the prevalence of these constraints on bnAb evolution may provide valuable insight for improving vaccination strategies (Yewdell, 2013; Henry et al., 2018).

To date, studies of antibody binding have been limited to small numbers of individual sequences, deep mutational scans of single mutations, and mutagenesis of small regions (Pappas et al., 2014; Braden et al., 1998; Burks et al., 1997; Adams et al., 2016; Koenig et al., 2017; Forsyth et al., 2013; Wu et al., 2017; Xu et al., 2015; Madan et al., 2021; Schmidt et al., 2015), due in part to practical constraints on library scale and the throughput of affinity assays. This has limited our ability to comprehensively characterize binding landscapes for naturally isolated bnAbs, which often involve many mutations spanning framework (FW) and complementarity-determining regions (CDR) (Corti et al., 2017; Corti and Lanzavecchia, 2013; Klein et al., 2013).

We overcome these challenges by generating combinatorially complete libraries of up to $\sim 10^{5}$ antibody sequences and assaying their binding affinities in a high-throughput yeast-display system (Adams et al., 2016). This approach enables us to infer the contributions of individual mutations as well as hundreds of pairwise and higher order interactions between mutations, revealing that these interactions can restrict evolutionary pathways leading to greater breadth. In particular, we find that mutational effects on binding affinity to diverse antigens display a nested structure, where increasingly large groups of specific mutations are required to gain affinity to divergent antigens, resulting in highly constrained paths to broad affinity. This pattern is not observed for more similar antigens, where many mutational paths to broad affinity are accessible. Further, these nested patterns of mutational effects provide new molecular insight into why sequential exposure to diverse antigens often favors greater breadth (Wang et al., 2010; Krammer et alı, 2012; Wang et alı, 2015; Wang, 2017; Sachdeva et al., 2020; Molari et alı, 2020; Sprenger et al., 2020). Together, this work provides the first comprehensive characterization of antibody affinity landscapes and advances our understanding of the molecular constraints on bnAb evolution. 


\section{A Antibody sequence alignment}

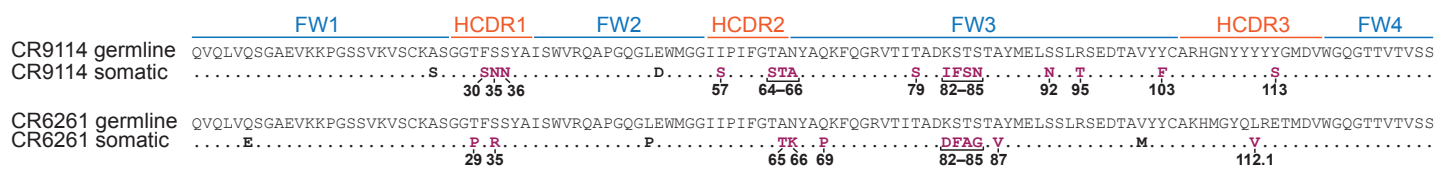

\section{CR9114 binding landscape}
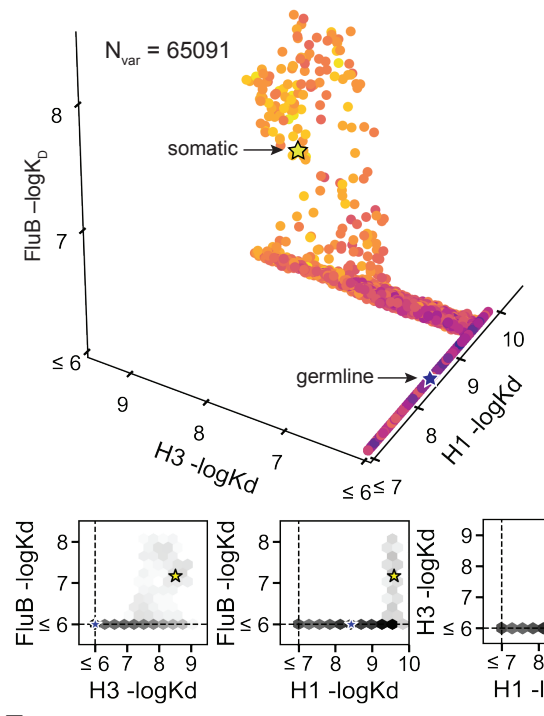

E
D

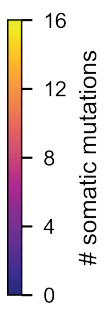

\section{CR9114 -logKd distribution by antigen}
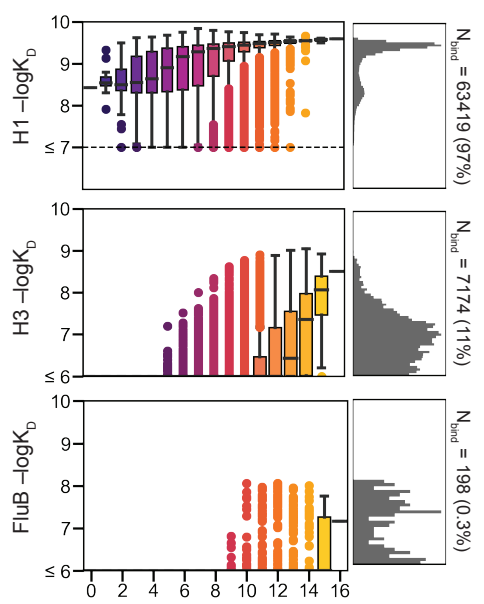

\# somatic mutations
B Influenza HA diversity $\&$ bnAb breadth

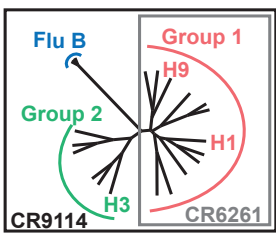

G CR9114 force-directed graph

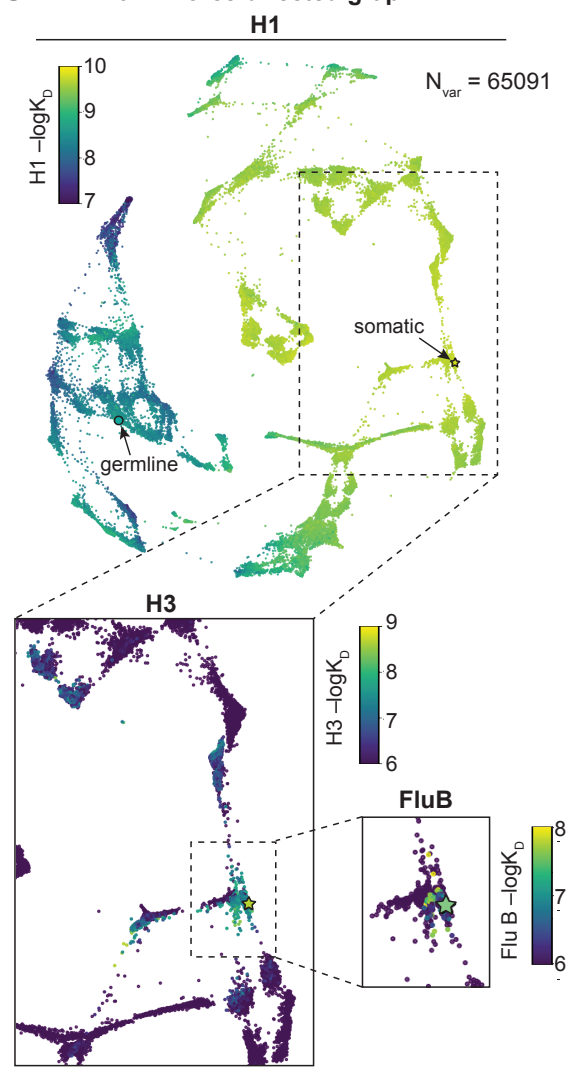

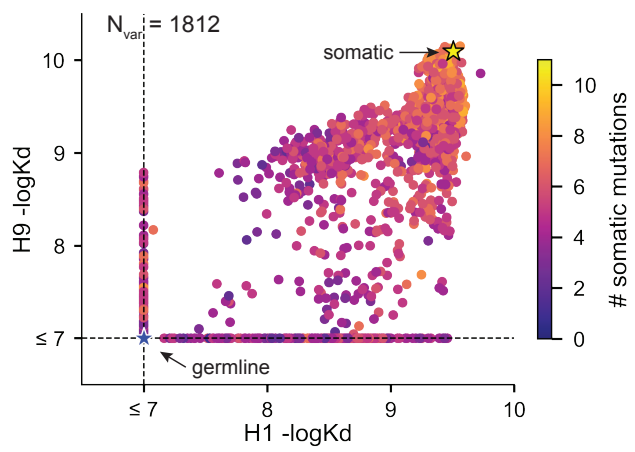
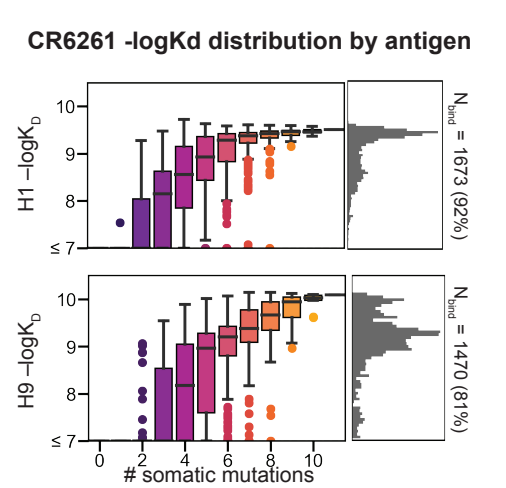

Figure 1. Binding landscapes. (A) Sequence alignment comparing somatic heavy chains to reconstructed germline sequences. Mutations under study (purple, numbered) and excluded mutations (black) are indicated; residues are numbered by IMGT unique numbering. (B) Influenza hemagglutinin phylogenetic tree with selected antigens and breadth of CR9114 (black box) and CR6261 (gray box) indicated. (C, E), Scatterplots of the (C) CR9114 library binding affinities against three antigens, with 2D planes shown below, and (E) CR6261 library binding affinities against two antigens. (D, F) Distributions of library binding affinities for (D) CR9114 and (F) CR6261 for each antigen (gray histogram, right) separated by number of somatic mutations (boxplots, left). Numbers and percentages of variants with measurable binding are indicated at right. (G), Force-directed graph of CR9114 H1 $-\log K_{D}$. Each variant (node) is connected to its 16 single-mutation neighbors (edges not shown for clarity); edges are weighted such that variants with similar genotypes and -log $K_{D}$ tend to cluster. Nodes are colored by binding affinity to H1 (top; showing all 65,091 nodes), H3 (lower left inset; showing only the region containing nodes with $-\log K_{D}>6$ ), and Flu B (lower right inset; showing only the region containing nodes with - log $K_{D}>6$ ).

The online version of this article includes the following source data and figure supplement(s) for figure 1 :

Source data 1. CR9114 library -log $K_{D}$ to $H 1, H 3$, and influenza B.

Source data 2. CR6261 library -log $K_{D}$ to $H 1$ and $H 9$.

Source data 3. Isogenic flow cytometry measurements of -log $K_{D}$ for select CR9114 and CR6261 variants.

Figure supplement 1. Experimental design and Tite-Seq workflow.

Figure supplement 2. Tite-Seq data quality.

Figure supplement 3. Antibody-antigen co-crystal structures.

Figure supplement 4. Force-directed graph for CR6261.

Figure 1 continued on next page 
Figure 1 continued

Figure supplement 5. Expression of antibody libraries.

Figure supplement $\mathbf{6}$. Tite-Seq gating strategy.

Figure supplement 7. Reversions of excluded mutations.

\section{Results}

\section{Binding affinity landscapes of CR9114 and CR6261}

Here, we characterize the binding affinity landscapes of the two well-studied bnAbs noted above: CR9114 and CR6261. Specifically, we made all combinations of a set of mutations separating the germline and somatic sequences for CR9114 (16 mutations totaling 65,536 variants) and CR6261 (11 mutations totaling 2048 variants). These libraries include all heavy-chain mutations in these antibodies, except a few select mutations distant from the paratope (Figure 1, Figure 1-figure supplement 7, and see Materials and methods). Both antibodies engage antigens solely through their heavy-chain regions (Dreyfus et al., 2012; Ekiert et al., 2009), and thus are well-suited for yeast display as single-chain variable fragments (see Materials and methods) (Boder and Wittrup, 1997).

We use the Tite-Seq method (Adams et al., 2016), which integrates flow cytometry and sequencing (Figure 1-figure supplement 1), to assay equilibrium binding affinities of each scFv sequence in these libraries against select antigens that span the breadth of binding for each antibody (Figure 1B). For CR6261, we chose two divergent group $1 \mathrm{HA}$ subtypes (H1 and H9; see Figure 1figure supplement 1), while for CR9114, we chose the three highly divergent subtypes present in the vaccine ( $\mathrm{H} 1$ from group 1, $\mathrm{H} 3$ from group 2, and influenza $\mathrm{B}$; see Figure 1-figure supplement 1, Throsby et al., 2008). Inferred affinities outside our titration boundaries $\left(10^{-11}-10^{-6} \mathrm{M}\right.$ for $\mathrm{H} 3$ and influenza $\mathrm{B}, 10^{-12}-10^{-7} \mathrm{M}$ for $\mathrm{H} 1$ and $\mathrm{H} 9$ ) are pinned to the boundary, as deviations beyond these boundaries are likely not physiologically relevant (Batista and Neuberger, 1998). Antibody expression is not strongly impacted by sequence identity, although some mutations have modest effects that may be inversely correlated with their effect on affinity (Figure 1-figure supplement 5). Affinities obtained by Tite-Seq are reproducible across biological triplicates (Figure 1-figure supplement 2; average standard error of $0.047-\log K_{D}$ units across antibody-antigen pairs) and are highly accurate as verified for select variants by isogenic flow cytometry (Figure 1-figure supplement 2) and by solution-based affinity measurements made by others (Throsby et al., 2008; Dreyfus et al., 2012; Lingwood et al., 2012; Pappas et al., 2014).

We begin by examining the distribution of binding affinities across antigens for each antibody library (Figure 1). We observe that most CR9114 variants have measurable affinity to H1 (97\%), fewer to $\mathrm{H3}(11 \%)$, and still fewer to influenza B $(0.3 \%)$ (Figure $\left.1 C_{1} D\right)$. For $\mathrm{H} 1$, only a few mutations are needed to improve from the germline affinity. In contrast, variants are not able to bind $\mathrm{H} 3$ unless they have several more mutations, and many more for influenza $\mathrm{B}$. This hierarchical structure is in striking contrast to the CR6261 library, in which most variants can bind both antigens $(92 \%$ for $\mathrm{H} 1$, $81 \%$ for $\mathrm{H} 9$ ), variants have a similar $\mathrm{K}_{\mathrm{D}}$ distribution, and many variants display intermediate affinity to both antigens (Figure 1E,F). To visualize how genotypes give rise to the hierarchical structure of CR9114 binding affinities, we represent the binding affinities for $\mathrm{H} 1$ as a force-directed graph. Here, each variant is a node connected to its 16 single-mutation neighbors, with edge weights inversely proportional to the change in $\mathrm{H} 1$ binding affinity, such that variants with similar genotype and $K_{D}$ tend to form clusters (Figure 1G, Figure 1-figure supplement 4). Coloring this genotype-to-phenotype map by the $-\log K_{D}$ to each of the three antigens, we see that sequences that bind $\mathrm{H} 3$ and influenza $B$ are highly localized and overlapping, meaning that they share specific mutations. Thus, while many CR9114 variants strongly bind $\mathrm{H} 1$, only a specific subset bind multiple antigens.

\section{Mutational effects on binding to diverse antigens}

To dissect how mutations drive the structure of these binding landscapes, we next infer specific mutational effects. We first log-transform binding affinities such that they are proportional to free energy changes $\left(\Delta G_{\text {binding }}\right)$, which should combine additively under the natural null expectation (Wells, 1990; Olson et al., 2014). We then define a linear model with single mutational effects and interaction terms up to a specified order (defined relative to the unmutated germline sequence, see 
Appendix 2 for alternatives), and fit coefficients by ordinary least squares regression. We use crossvalidation to identify the maximal order of interaction for each antigen and report coefficients at each order from these best-fitting models (CR9114: fifth order for H1, fourth for H3, first for influenza B; CR6261: fourth order for $\mathrm{H} 1$ and $\mathrm{H} 9$; see Materials and methods). We note that the maximum order of interactions is affected by our inference power, particularly by the number of sequences with appreciable binding, and so we interpret these models as showing strong evidence of epistasis at least up to the order indicated. We explored the possibility of 'global' epistasis by inferring a nonlinear transformation of the $-\log K_{D}$ values (Sailer and Harms, 2017a; Otwinowski et al., 2018), but found that this approach did not significantly reduce the order or number of specific interaction coefficients needed to explain the data (see Appendix 2). We also explored inferring epistasis up to full order using Walsh-Hadamard transformations; results are qualitatively similar but less conservative than cross-validated regression (see Appendix 2).

Examining the effect of individual mutations on the germline background (Figure 2A,B), we observe several mutations that enhance binding to all antigens (e.g. S83F for CR9114), and mutations that confer trade-offs for binding distinct antigens (e.g. F30S in CR9114 reduces affinity for $\mathrm{H} 1$ but enhances affinity for influenza B). Generally, large-effect mutations are at sites that contact HA (Figure 2C, Figure 2-figure supplement 1, Dreyfus et al., 2012; Ekiert et al., 2009). Consistent with prior biochemical and structural work, mutations essential for CR9114 breadth are spread throughout FW3 and the CDRs, forming hydrophobic contacts and hydrogen bonds with residues in the conserved HA stem epitope (Dreyfus et alo, 2012; Avnir et alo, 2014). We observe three specific mutations that are required for binding to $\mathrm{H} 3$ (present at over $90 \%$ frequency in the set of binding sequences), likely because they form hydrophobic contacts with HA (K82I and S83F) and reorient the CDR2 loop (I57S), which interacts with residues and a glycan in $\mathrm{H} 3$ that are distinct from those in $\mathrm{H} 1$ (Dreyfus et al., 2012). We also observe eight specific mutations that are required for binding to influenza B. Many of these breadth-conferring mutations are absent in CR6261, particularly those in CDR2 (Dreyfus et alo, 2012; Ekiert et alo, 2009). Notably, these sets of required mutations in CR9114 exhibit a nested structure: mutations beneficial for $\mathrm{H} 1$ are required for $\mathrm{H} 3$, and mutations required for $\mathrm{H} 3$ are required for influenza $\mathrm{B}$, giving rise to the hierarchical structure of the binding landscape (Figure 1C).

Beyond these exceptionally synergistic interactions between required mutations, we find that epistasis is widespread, accounting for $18-33 \%$ of explained variance depending on the antibodyantigen pair (except influenza B, see Materials and methods, Appendix 2). Pairwise interactions are dominated by a few mutations (e.g. F30S for CR9114 and S35R for CR6261) that exhibit many interactions, both positive and negative, with other mutations (Figure 2D,E). Overall, mutations with strong pairwise interactions tend to be close in the crystal structure, although there are long-range pairwise interactions that are likely mediated by interactions with the antigen or conformational rearrangements (Figure 2F, Figure 2-figure supplement 1, Dreyfus et alo, 2012; Ekiert et al., 2009; Avnir et al., 2014).

\section{High-order epistasis is dominated by a subset of mutations}

Our dataset also allows us to resolve higher order epistasis. In addition to the required mutations, our models identify numerous strong third to fifth order interactions, with a subset of mutations participating in many mutual interactions at all orders. For CR9114 binding to H1, this subset consists of five mutations, distributed across three different regions of the heavy chain (Figure 3A,B). Some of these mutations likely generate (K82I, S83F) or abrogate (F30S) contacts to HA, and others (I57S, A65T) may indirectly impact HA binding by reorienting contact residues in CDR2 (Dreyfus et alo, 2012; Avnir et al., 2014). Within this set of five residues, we first illustrate two examples of thirdorder epistasis by grouping sequences by their genotypes at these five sites (Figure 3C). Intriguingly, some mutations that are deleterious in the germline background (' - ' annotations) are beneficial in doubly-mutated backgrounds (' + ' annotations). For example, mutation F30S is significantly less deleterious in backgrounds with S83F than in the germline background, suggesting that new hydrophobic contacts in FW3 may be able to compensate for the potential loss of contacts in CDR1. Yet F30S unexpectedly becomes beneficial after an additional mutation I57S in CDR2, indicating more complex interactions between flexible CDR and FW loop regions (Figure 3B,C; Dreyfus et al。, 2012). 


\section{A CR9114: First order effects}

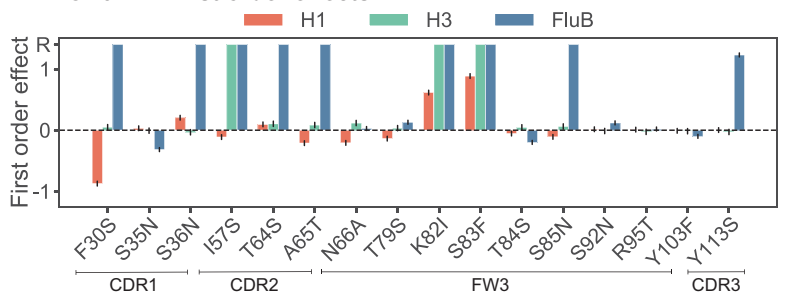

B CR6261: First order effects

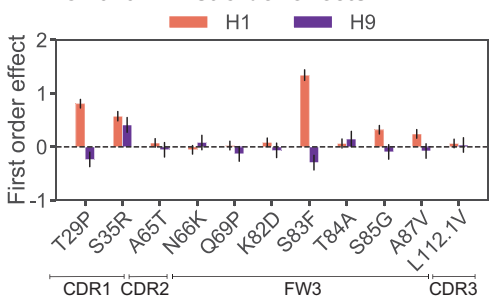

C First order effects vs. HA contact surface area

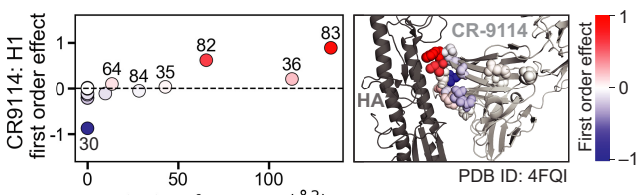

contact surface area $\left(\AA^{2}\right)$

D CR9114: Second order effects

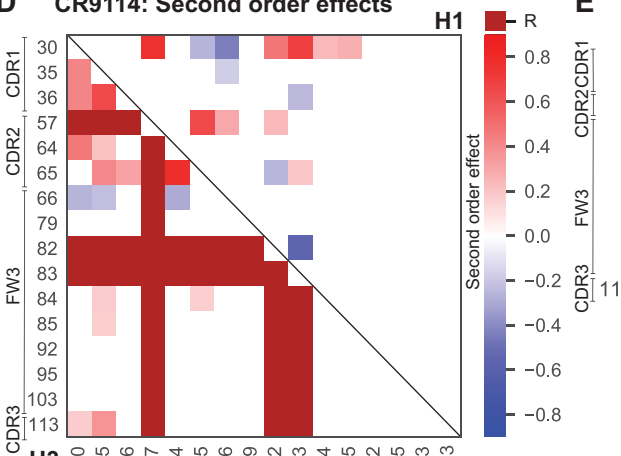

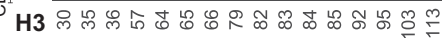

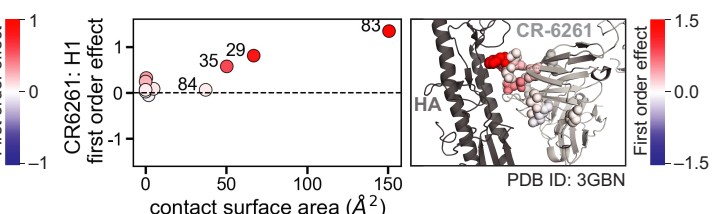

E CR6261: Second order effects

F Second order effects

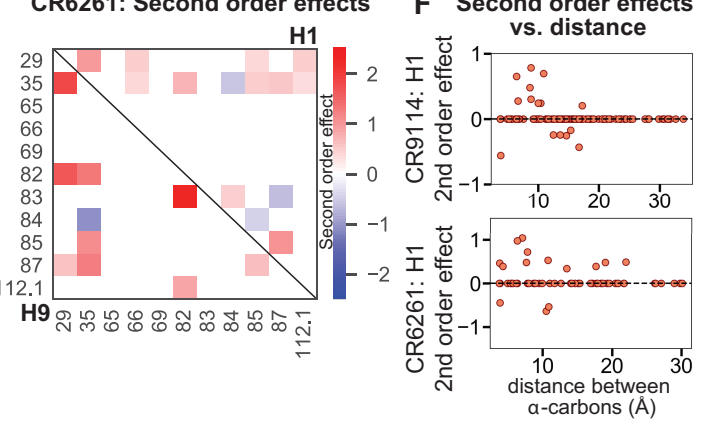

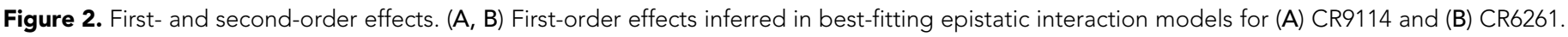

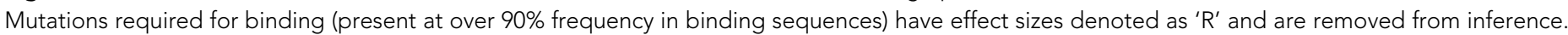
Error bars indicate standard error. (C) First order effects for each site plotted against the contact surface area between the corresponding somatic

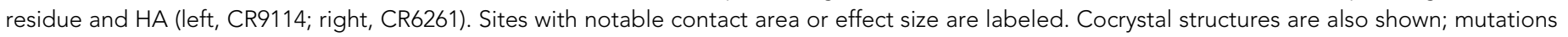

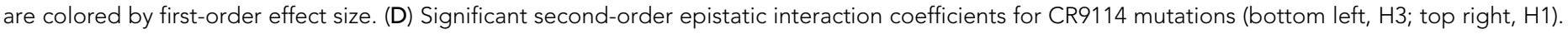

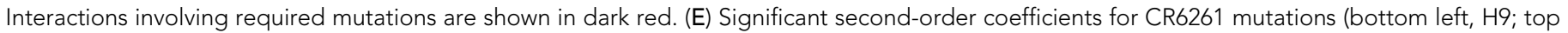

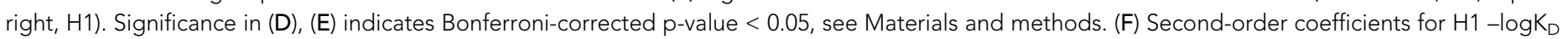
plotted against the distance between the respective $\alpha$-carbons in the crystal structures.

The online version of this article includes the following source data and figure supplement(s) for figure 2 :

Source data 1. Interaction model coefficients for CR9114.

Source data 2. Interaction model coefficients for CR6261.

Source data 3. Tabulated contact surface area, number of HA contacts, and pairwise distances for mutations in CR9114 and CR6261.

Figure supplement 1 . Structural context of first and second order effects.

To see how these high-order interactions drive the overall structure of the binding affinity landscape, we return to the force-directed graph, now colored by genotype at these five key sites (Figure 3D; only points corresponding to genotypes shown in Figure 3C are colored). We see that these five sites largely determine the overall structure of the map: points of the same color tend to cluster together, despite varying in their genotypes at the other 11 sites. However, we observe that interactions with other mutations do exist, as evidenced by separate clusters with the same color (e.g. the two clusters in teal for 57,65 are distinguished by a positive third-order interaction with site 64, Figure 3E). These patterns are not confined to the genotypes shown in Figure 3C; if we color all 32 possible genotypes at the five key sites, we observe the same general patterns (Figure 3-figure supplement 1; an interactive data browser for exploring these patterns of epistasis in CR9114 is available at: https://yodabrowser.netlify.app/yoda_browser/). Interactions between these five sites are also enriched for significant epistatic coefficients $\left(p<10^{-3} ; 26\right.$ of 31 possible terms are significant, 
A CR9114 H1: $3^{\text {rd }}-5^{\text {th }}$ order effects

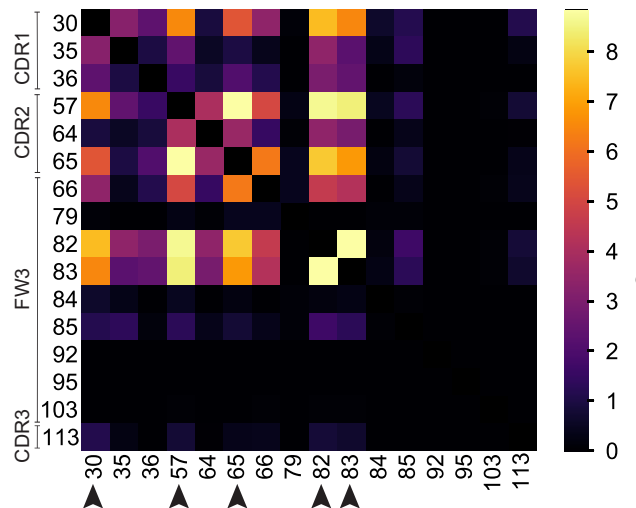

C CR9114 H1: high-order interactions between $30,57,65,82, \& 83$

- additive expectation …... pairwise expectation

Mutations F30S, 157S, S83F (with A65 \& K82)

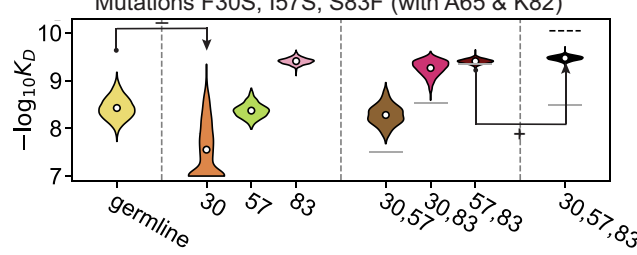

Mutations I57S, A65T, K82I (with F30 \& S83)

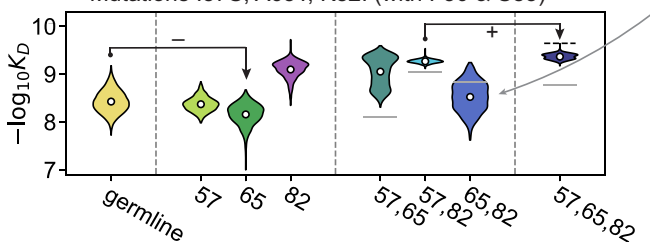

$\mathbf{F}$

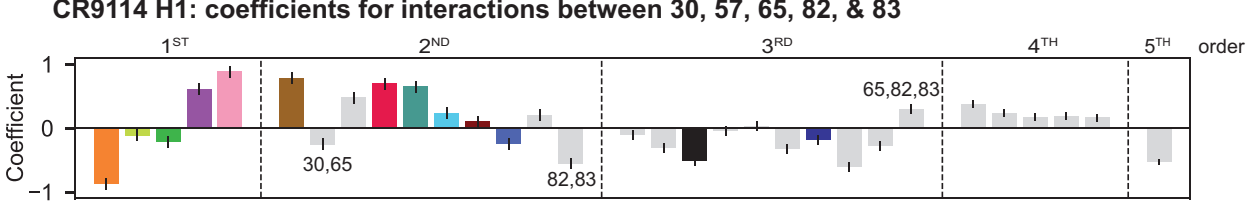

B CR9114 H1: location of key epistatic residues

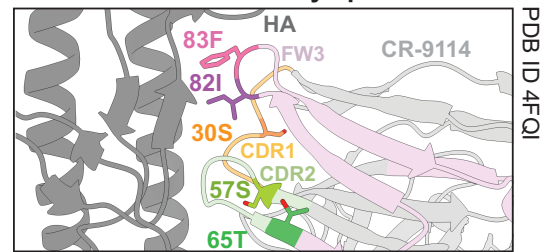

D CR9114 H1: high-order interaction map
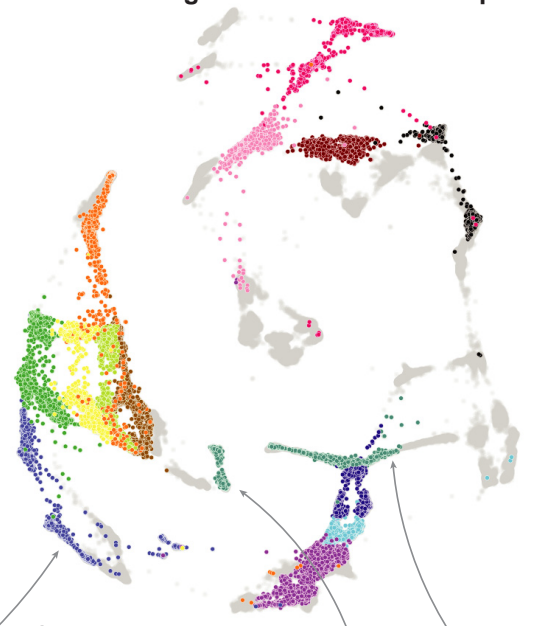

E CR9114 H1: other high-order interactions Mutations 157S, T64S, A65T (with F30, K82 \& S83)

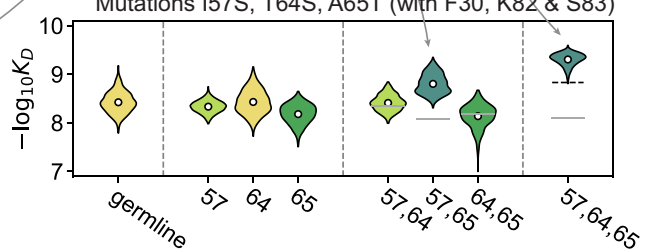

(n)


compared to an average of 4 terms among all sets of five sites, Figure 3-figure supplement 1), including the fifth order interaction between all five residues (Figure 3F). Remarkably, these five mutations underlie significant high-order epistasis for other antigens as well: all five are either required for binding or participate extensively in interactions for $\mathrm{H} 3$ and influenza B (Figure 3-figure supplement 3 ).

Higher order epistasis in CR6261 is similarly dominated by a subset of mutations in CDR1 and FW3, at identical or neighboring positions as some key sites for CR9114 (Figure 4A). These mutations exhibit strong diminishing returns epistasis at third and fourth order, counteracting their synergistic pairwise effects, in a similar manner across both antigens (Figure 4B, Figure 4-figure supplement 1, Figure 4-figure supplement 2). Many fourth-order combinations of these mutations display interaction coefficients of similar magnitude (Figure 4-figure supplement 1), though they may be signatures of even higher order interactions that we are underpowered to infer.

A common approach to quantify how epistasis constrains mutational trajectories is to count 'uphill' paths (i.e. where affinity improves at every mutational step from the germline to the somatic sequence). We find that only a small fraction of potential paths are uphill $(0.00005 \%+/-0.00004 \%$ for CR9114 binding $\mathrm{H} 1$, and $0.2 \%+/-0.04 \%$ for CR6261 binding $\mathrm{H} 1$, as estimated by bootstrap, see Materials and methods). However, we note that for all antibody-antigen combinations, the somatic sequence is not the global maximum of the landscape (the best-binding sequence) and some mutations have deleterious effects on average. Hence, strictly uphill paths are only possible due to sign epistasis, where normally deleterious mutations have beneficial effects in specific genetic backgrounds.

Overall, we see that mutational effects and interactions between them explain the affinity landscapes we observe. For CR9114, binding affinity to H1 can be achieved through different sets of few mutations with complex interactions. In contrast, a specific set of many mutations with strong synergistic interactions is required to bind $\mathrm{H} 3$, and to an even greater extent, influenza B (Figure 2A), giving rise to the landscape's hierarchical structure (Figure 1C). For CR6261, the higher order interactions are more similar between $\mathrm{H} 1$ and $\mathrm{H} 9$, which is consistent with the more correlated patterns of binding affinities between these two antigens (Figure 1E).

\section{Affinity to diverse antigens was likely acquired sequentially}

The hierarchical nature of the CR9114 landscape suggests that this lineage developed affinity to each antigen sequentially. Considering the maximum $-\log K_{D}$ achieved by sequences with a given

A CR6261 H1: $3^{\text {rd }}-4^{\text {th }}$ order effects

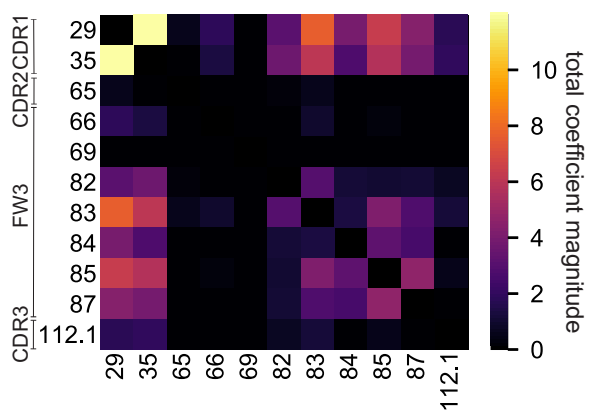

B CR6261 H1: $3^{\text {rd }}$ order interaction

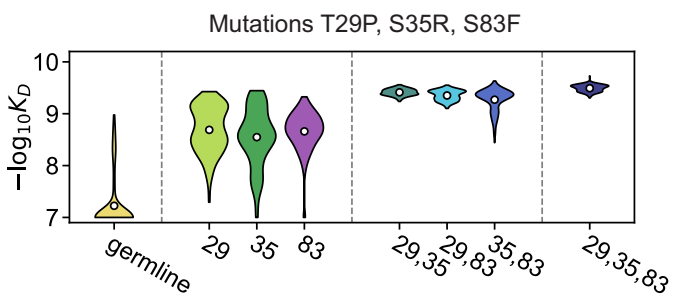

Figure 4. High-order epistasis for CR6261. (A) Total significant epistatic contributions of CR6261 mutation pairs for binding $\mathrm{H1}$, as in Figure 3A. Significance is given by Bonferroni-corrected p-value $<0.05$, see Materials and methods. (B) Third-order interaction for CR6261 H1 binding between mutations T29P, S35R, and S83F $(N=256$ genotypes per violin).

The online version of this article includes the following figure supplement(s) for figure 4:

Figure supplement 1. CR6261: interactions between four sites.

Figure supplement 2. High-order epistasis for CR6261 binding to H9. 
number of mutations (a proxy for time), we see that improvements in $\mathrm{H} 1$ binding can be realized early on, whereas improvements in $\mathrm{H} 3$ binding are not possible until later, and even later for influenza B (Figure 5A). In fact, the nested structure of affinity-enhancing mutations forces improvements in binding affinity to occur sequentially. If selection pressures were also experienced in this sequence, mutations that improve binding to the current antigen would lead to the genotypes required to begin improving binding to the next. Indeed, we find that for CR9114, there are more uphill paths leading to the somatic sequence if selection acts first on binding to $\mathrm{H} 1$ and later to $\mathrm{H} 3$ and influenza B (Figure 5C). In contrast, for CR6261, improvements in binding can occur early on for

A CR9114: Maximum -logK

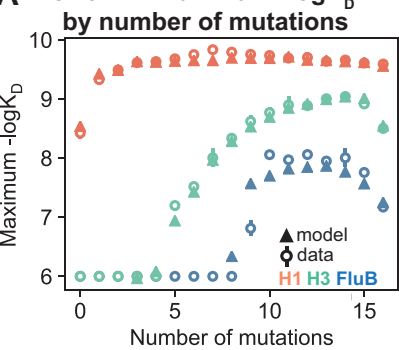

C CR9114: Number of uphill paths

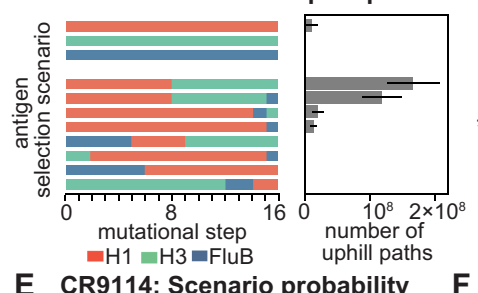

CR9114: Scenario probability

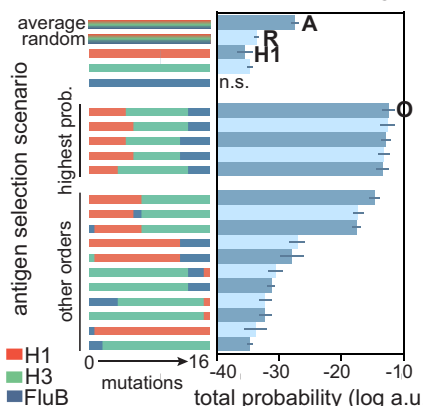

B CR6261: Maximum -logK by number of mutations

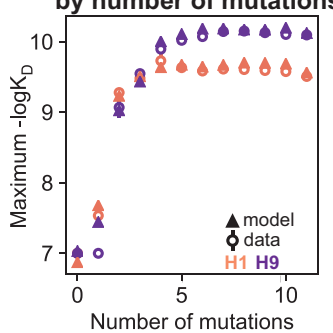

D CR6261: Number of uphill paths

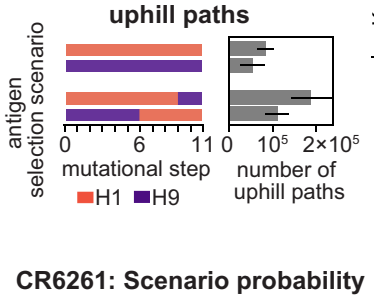

CR6261: Scenario probability

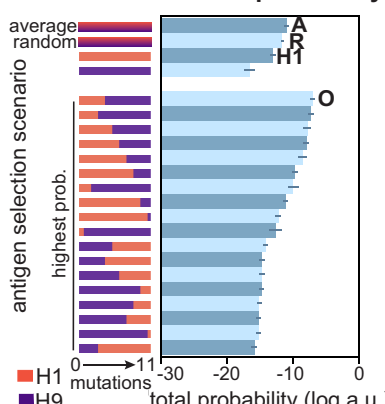

G CR9114: Most likely paths for selection scenarios $\mathrm{O}, \mathrm{A}, \mathrm{R}, \mathrm{H1}$ :
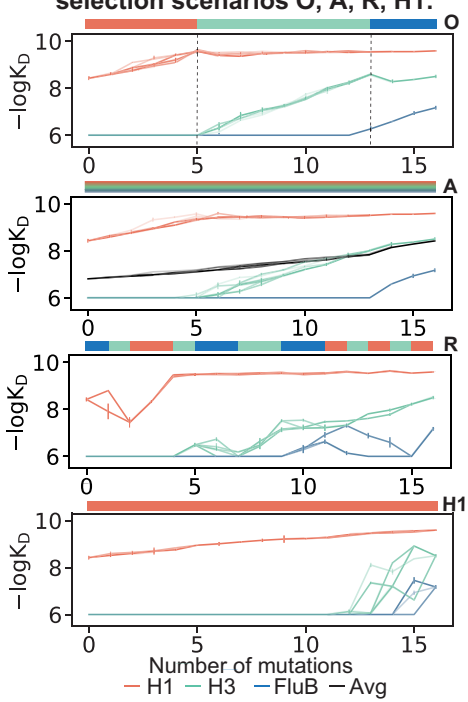

I CR9114: Mutation order under

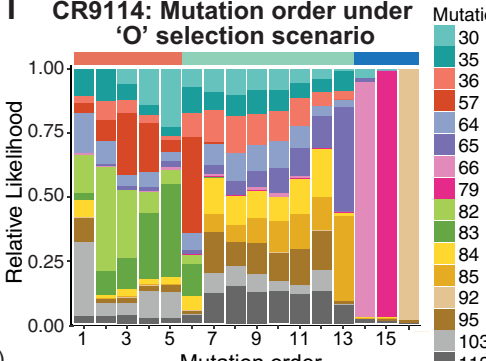

H CR6261: Most likely paths for selection scenarios $\mathrm{O}, \mathrm{A}, \mathrm{R}, \mathrm{H1}$ :
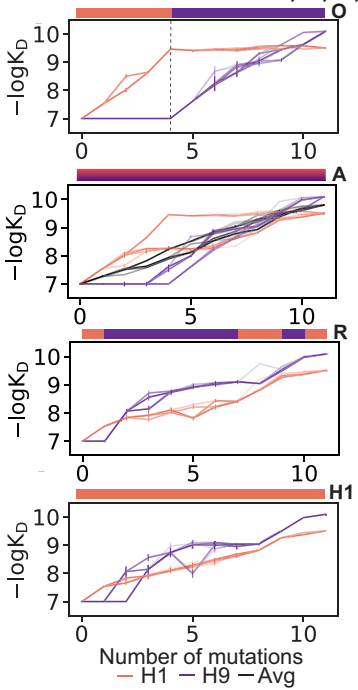

J CR6261: Mutation order under

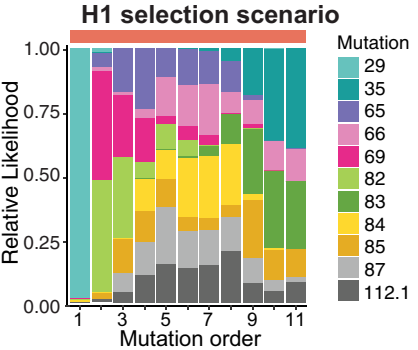

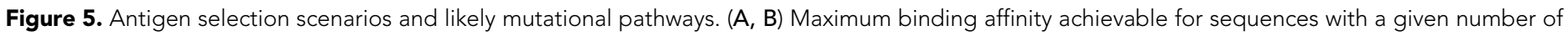

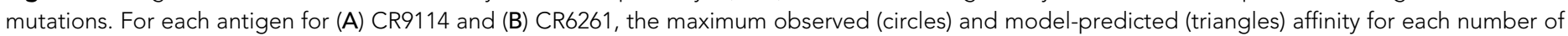

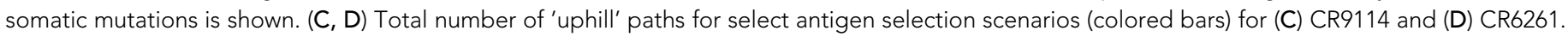

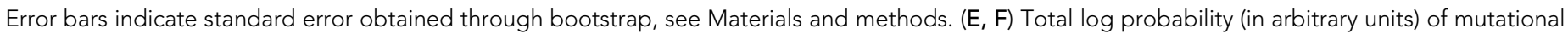
trajectories from germline to somatic sequence for (E) CR9114 and (F) CR6261 under different antigen selection scenarios, in a moderate selection

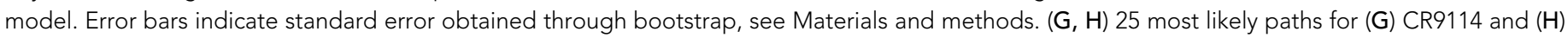
CR6261, from select scenarios in (E, F); -logK $K_{D}$ plotted for each antigen. For the random mixed scenario ('R'), a representative case is shown. 'A'

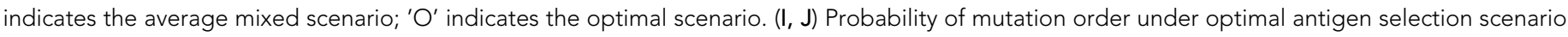

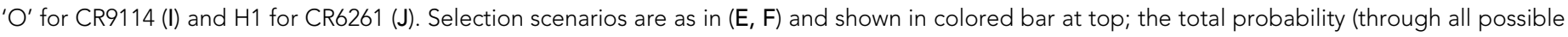
paths) for each mutation to occur at each mutational step is shown as stacked colored bars.

The online version of this article includes the following source data and figure supplement(s) for figure 5 :

Source data 1. Total probability of mutational trajectories for CR9114 under different antigen selection scenarios.

Source data 2. Total probability of mutational trajectories for CR6261 under different antigen selection scenarios.

Figure supplement 1. Selection models.

Figure supplement 2. Variant probabilities for CR9114 under the optimal ('O') selection model.

Figure supplement 3. Probability of mutation order assuming moderate selection, under other antigen selection scenarios. 
both antigens (Figure 5B) and the number of uphill paths is more similar across single-antigen and sequential selection pressures (Figure 5D).

To compare antigen selection scenarios more generally, we developed a framework that evaluates the total probability of all possible mutational pathways from germline to somatic, under an array of antigen selection scenarios (individual, sequential, and mixed). Our framework assumes that the probability of any mutational step is higher if - log $K_{D}$ increases, but does not necessarily forbid neutral or deleterious steps; we evaluate a variety of specific forms of this step probability and find that our major results are consistent (Figure 5-figure supplement 1A, see Materials and methods). We assume that each amino acid substitution occurs in a single mutational step; though there are amino acid substitutions that must proceed by multiple nucleotide mutations that may occur in a single round, or over multiple rounds, of somatic hypermutation (Spisak et al., 2020; Unniraman and Schatz, 2007). Mixed antigen regimes approximate exposure to a cocktail of antigens. We model these with two approaches: (1) 'average', using the average -log $K_{D}$ across all antigens, and (2) 'random', using $-\log K_{D}$ for a randomly selected antigen at each step (note that using the maximum $\log K_{D}$ across antigens would always be trivially favored) (Wang et al., 2015). While these models simplify the complexities of affinity maturation in vivo (Victora and Nussenzweig, 2012), especially how affinity relates to $B$ cell lineage dynamics and the mutational bias at the nucleotide level (Spisak et al., 2020), they provide insight into the relative probabilities of mutational paths under distinct antigen selection scenarios.

Again we find that the vast majority of likely antigen selection scenarios for CR9114 involve first $\mathrm{H} 1$, followed by H3, followed by influenza B (Figure 5E, Figure 5-figure supplement 1B). These results are underscored by examining improvement in $-\log \mathrm{K}_{\mathrm{D}}$ along the most likely mutational paths for each scenario (Figure 5G): in the optimal sequential scenario, -log $K_{D}$ can improve substantially for each antigen in turn, while in an $\mathrm{H} 1$-only scenario, the improvements in $\mathrm{H} 1$ binding at each step are much more gradual, reducing the likelihood. The average mixed scenario shows qualitatively similar paths to the optimal sequential scenario, although with lower overall probability. In the random mixed scenario, even the best pathways are often unable to improve affinity to the randomly selected antigen, and affinity to antigens not under selection often declines, making these scenarios much less likely.

Given the optimal sequential selection scenario, the vast majority of genotypes are unlikely evolutionary intermediates to the somatic sequence (Figure 5-figure supplement 2). We visualize the impact of epistasis on mutational order by considering the probability of each mutation to occur at each mutational step (Figure 5I; Figure 5-figure supplement 3). The three antigen exposure epochs exhibit clear differences in favored mutations. Mutations I57S, K82I, and S83F must occur early, due to their strong synergistic interactions for all three antigens. In addition, we see that F30S is unlikely to happen very early (due to its sign epistasis under $\mathrm{H} 1$ selection) as well as unlikely to happen very late (due to its strong benefit under influenza B selection).

In contrast, for CR6261, all selection scenarios have relatively similar likelihood (Figure 5F, Figure 5-figure supplement 1C). Among sequential scenarios, however, those beginning with $\mathrm{H} 1$ are more likely than those beginning with $\mathrm{H}$, as the first two mutational steps can improve affinity to $\mathrm{H} 1$ more than $\mathrm{H} 9$, and mutations late in maturation can improve affinity to $\mathrm{H} 9$ more than $\mathrm{H} 1$ (Figure 1F, Figure 5B). Still, unlike CR9114, in both single antigen and mixed scenarios, there are many likely paths that continually improve in binding to both antigens (Figure $5 \mathbf{H}$ ). Initially the order of mutations is highly constrained due to strong synergistic epistasis, and differences between selection scenarios reflect differences in mutational effects between antigens (Figure 5J, Figure 5-figure supplement 3). We note that T29P is highly likely to occur first in scenarios that begin with $\mathrm{H} 1$, as this is the only single mutation that can improve $\mathrm{H} 1$ affinity, albeit rather modestly.

\section{Discussion}

Overall, we find that evolutionary pathways to bnAbs can be highly contingent on epistatic and pleiotropic effects of mutations. Specifically, the acquisition of breadth for CR9114 is extremely constrained and is likely to have occurred through exposure to diverse antigens in a specific order, due to the structure of correlations and interactions between mutational effects. In contrast, CR6261 could have acquired affinity to $\mathrm{H} 1$ and $\mathrm{H} 9$ in a continuous and simultaneous manner, perhaps 
because these antigens are more similar; since $\mathrm{H} 9$ is not a commonly circulating strain, this breadth was likely acquired by chance (Pappas et al., 2014).

We note that we cannot conclusively determine how CR9114 and CR6261 evolved in vivo. The isolation of these specific antibodies from phage display libraries (Throsby et al., 2008; Dreyfus et al., 2012) was likely biased by the HA subtypes used for screening, and although unlikely, may have introduced mutations during PCR amplification. Regardless, these antibody sequences occupy regions of sequence space that are useful for understanding the relationship between sequence, affinity, and breadth. By characterizing their binding landscapes, we find that epistasis and trade-offs constrain the mutational pathways to these specific somatic sequences and their associated breadth. Indeed, we find that not all of the observed mutations are required to confer broad affinity, and future work is needed to explore what alternative pathways to breadth might be accessible through other mutations. It is also worth noting that selection pressure to bind the HA stem epitope on virions may be different from pressure to bind soluble recombinant HA, although several studies have found anti-stem antibody affinity to recombinant $\mathrm{HA}$ to be indicative of viral neutralization (Dreyfus et al., 2012; Corti et al., 2011; Lingwood et al., 2012). Further, stem-targeting bnAbs and their germline precursors have been characterized as polyreactive (Bajic et al., 2019; Guthmiller et al., 2020) and thus likely experience additional selection pressures that are not captured by our measurements and models, such as negative selection against autoreactivity. Although we cannot determine which specific antigens were involved in the selection of these antibodies in vivo, the diverse HA subtypes we employ capture variation representative of circulating influenza strains and thus serve as useful probes of varying levels of breadth (Corti et al., 2017). Future work integrating these measurements of affinity and breadth with measurements of stability and polyreactivity will provide important insight into the molecular constraints of bnAb evolution.

Notably, the landscapes characterized here are among the largest combinatorially complete collections of mutations published to date. In some respects, our observations of high-order interactions are consistent with earlier work in other proteins. In particular, epistasis has been found to affect function and constrain evolutionarily accessible pathways across functionally and structurally distinct proteins (Weinreich et al., 2006; Starr et al., 2017; Ortlund et al., 2007; Podgornaia and Laub, 2015; Gong et alı, 2013; Sailer and Harms, 2017b; Miton and Tokuriki, 2016; Poelwijk et al., 2019; Bank et al., 2015). Further, pairwise and high-order epistasis appear to be common features of binding interfaces, such as enzyme-substrate and receptor-ligand interactions (Weinreich et al., 2006; Starr et al., 2017; Ortlund et al., 2007; Podgornaia and Laub, 2015; Sailer and Harms, 2017b; Miton and Tokuriki, 2016), and interacting mutations are often spaced in both sequence and structure, underscoring the complexity of protein-protein interfaces (Podgornaia and Laub, 2015; Adams et al., 2019; Braden et al., 1998; Esmaielbeiki et al., 2016; Rotem et al., 2018). On the other hand, the strongly synergistic, nested mutations crucial for CR9114 breadth are unusual, perhaps due to the nature of antibody-antigen interfaces or to the unique dynamics of affinity maturation (Victora and Nussenzweig, 2012). Together, these observations suggest that interactions between multiple mutations, such as those we characterize here, could play a substantial role in affinity maturation and may contribute to the rarity of bnAbs in natural repertoires.

Our findings provide molecular insight into the emerging picture of how selection can elicit broad affinity, illustrated by a substantial recent body of work ranging from in vivo experimental approaches (Krammer et al., 2012; Wang et al., 2010) to quantitative modeling of immune system dynamics (Wang et al., 2015; Wang, 2017; Sachdeva et al., 2020; Molari et al., 2020; Sprenger et al., 2020). These diverse studies often find that mixed-antigen regimens are less effective than sequential regimens at eliciting bnAbs. Our results demonstrate that, at least in part, this may be due to the intrinsic structure of the mutational landscape, defined by the complex interactions of mutational effects across antigens. With more studies of binding landscapes for diverse antibodies, we could better understand how such features generalize between different germline sequences, somatic mutation profiles, and antigen molecules. These insights will be valuable for leveraging germline sequence data and antigen exposure information to predict, design, and elicit bnAbs for therapeutic and immunization applications. 


\section{Materials and methods}

\section{Key resources table}

\begin{tabular}{|c|c|c|c|c|}
\hline $\begin{array}{l}\text { Reagent type } \\
\text { (species) or resource }\end{array}$ & Designation & Source or reference & Identifiers & Additional information \\
\hline $\begin{array}{l}\text { Strain, strain } \\
\text { background } \\
\text { (Saccharomyces } \\
\text { cerevisiae) }\end{array}$ & EBY100 & ATCC & Cat\#:MYA-4941 & \\
\hline $\begin{array}{l}\text { Cell line } \\
\text { (Spodoptera } \\
\text { frugiperda) }\end{array}$ & Sf9 & ThermoFisher & Cat\#:B82501 & $\begin{array}{l}\text { Cell line for production } \\
\text { of baculovirus }\end{array}$ \\
\hline $\begin{array}{l}\text { Cell line } \\
\text { (Trichoplusia ni) }\end{array}$ & High-Five & ThermoFisher & Cat\#:B85502 & $\begin{array}{l}\text { Cell line for HA } \\
\text { expression }\end{array}$ \\
\hline Antibody & $\begin{array}{l}\text { Anti-cMyc-FITC } \\
\text { (Mouse monoclonal) }\end{array}$ & Miltenyi Biotec & Cat\#:130-116-485 & FACS $(1: 50)$ \\
\hline $\begin{array}{l}\text { Recombinant } \\
\text { DNA reagent }\end{array}$ & рCT302 (plasmid) & Addgene & Cat\#:41845 & \\
\hline $\begin{array}{l}\text { Recombinant } \\
\text { DNA reagent }\end{array}$ & $\begin{array}{l}\text { PCT302_CR9114 } \\
\text { _germline (plasmid) }\end{array}$ & This paper & & $\begin{array}{l}\text { Plasmid map in } \\
\text { Supplementary file } 4\end{array}$ \\
\hline $\begin{array}{l}\text { Recombinant } \\
\text { DNA reagent }\end{array}$ & $\begin{array}{l}\text { PCT302_CR9114 } \\
\text { somatic (plasmid) }\end{array}$ & This paper & & $\begin{array}{l}\text { Plasmid map in } \\
\text { Supplementary file } 5\end{array}$ \\
\hline $\begin{array}{l}\text { Recombinant } \\
\text { DNA reagent }\end{array}$ & $\begin{array}{l}\text { PCT302_CR6261 } \\
\text { _germline (plasmid) }\end{array}$ & This paper & & $\begin{array}{l}\text { Plasmid map in } \\
\text { Supplementary file } 6\end{array}$ \\
\hline $\begin{array}{l}\text { Recombinant } \\
\text { DNA reagent }\end{array}$ & $\begin{array}{l}\text { PCT302_CR6261 } \\
\text { _somatic (plasmid) }\end{array}$ & This paper & & $\begin{array}{l}\text { Plasmid map in } \\
\text { Supplementary file } 7\end{array}$ \\
\hline $\begin{array}{l}\text { Recombinant } \\
\text { DNA reagent }\end{array}$ & pET21a-BirA (plasmid) & Addgene & Cat\#:20857 & \\
\hline $\begin{array}{l}\text { Sequence- } \\
\text { based reagent }\end{array}$ & $\begin{array}{l}\text { CR9114 golden gate } \\
\text { dsDNA fragments }\end{array}$ & IDT & & $\begin{array}{l}\text { Sequences listed in } \\
\text { Supplementary file } 2\end{array}$ \\
\hline $\begin{array}{l}\text { Sequence- } \\
\text { based reagent }\end{array}$ & $\begin{array}{l}\text { CR6261 Golden } \\
\text { Gate primers }\end{array}$ & IDT & & $\begin{array}{l}\text { Sequences listed in } \\
\text { Supplementary file } 3\end{array}$ \\
\hline $\begin{array}{l}\text { Sequence- } \\
\text { based reagent }\end{array}$ & $\begin{array}{l}\text { Illumina } \\
\text { sequencing } \\
\text { primers }\end{array}$ & IDT & & $\begin{array}{l}\text { Sequences listed in } \\
\text { Supplementary file } 1\end{array}$ \\
\hline $\begin{array}{l}\text { Peptide, } \\
\text { recombinant } \\
\text { protein }\end{array}$ & Streptavidin-RPE & ThermoFisher & Cat\#:S866 & FACS $(1: 100)$ \\
\hline $\begin{array}{l}\text { Peptide, } \\
\text { recombinant } \\
\text { protein }\end{array}$ & $\begin{array}{l}\text { Biotinylated A/New } \\
\text { Caledonia/99 }(\mathrm{H} 1) \\
\text { ectodomain }\end{array}$ & This paper & & $\begin{array}{l}\text { Plasmid sequence in } \\
\text { Supplementary file } 8\end{array}$ \\
\hline $\begin{array}{l}\text { Peptide, } \\
\text { recombinant } \\
\text { protein }\end{array}$ & $\begin{array}{l}\text { Biotinylated A/Hong Kong/99 } \\
\text { (H9) ectodomain }\end{array}$ & This paper & & $\begin{array}{l}\text { Plasmid sequence in } \\
\text { Supplementary file } 9\end{array}$ \\
\hline $\begin{array}{l}\text { Peptide, } \\
\text { recombinant } \\
\text { protein }\end{array}$ & $\begin{array}{l}\text { Biotinylated A/Wisconsin/05 } \\
(\mathrm{H} 3) \text { ectodomain }\end{array}$ & This paper & & $\begin{array}{l}\text { Plasmid sequence in } \\
\text { Supplementary file } 10\end{array}$ \\
\hline $\begin{array}{l}\text { Peptide, } \\
\text { recombinant } \\
\text { protein }\end{array}$ & $\begin{array}{l}\text { Biotinylated B/ } \\
\text { Ohio/05 (Flu B) } \\
\text { ectodomain }\end{array}$ & This paper & & $\begin{array}{l}\text { Plasmid sequence in } \\
\text { Supplementary file } 11\end{array}$ \\
\hline $\begin{array}{l}\text { Commercial } \\
\text { assay or kit }\end{array}$ & Bac-to-Bac Kit & ThermoFisher & Cat\#:10359016 & \\
\hline $\begin{array}{l}\text { Commercial } \\
\text { assay or kit }\end{array}$ & $\begin{array}{l}\text { Zymo Yeast } \\
\text { Plasmid Miniprep II }\end{array}$ & Zymo Research & Cat\#:D2004 & \\
\hline
\end{tabular}

Continued on next page 
Continued

Reagent type (species) or resource

Software,

algorithm
Source or reference

This paper
Identifiers

Custom code
Additional information

https://github.com/

klawrence26/bnab-landscapes (copy archived at swh:1:rev:61c1673a101 ea739d5b7e9b282fb bcfad41d7e90, Phillips, 2021)

Software, algorithm
Interactive CR9114 data browser

This paper https://yodabrowser.

netlify.

app/yoda_browser/

\section{Antibody library production}

Germline sequence reconstructions

For CR9114, we obtained the somatic heavy chain nucleotide sequence from Dreyfus et al., 2012 (GenBank JX213639.1) and reconstructed the germline nucleotide sequence using IMGT (Giudicelli et al., 2006) and IgBLAST (Ye et al., 2013). Both methods assigned the same V-gene and J-gene alleles (IGHV1-69*06 and IGHJ6*02), but there is ambiguity in the D-gene assignment and at the V-D junction, particularly at site 109. The preferred IMGT junction alignment assigns a mutation here, S109N, while a different junction alignment from IgBLAST does not. Because of the inherent difficulty of reconstructing mutations in the junction region, especially in antibodies with a short $D$ region, we chose the alignment without the mutation at site 109 . Our reconstructed germline nucleotide sequence is available in Supplementary file 12. We then took the resulting germline and somatic amino acid sequences, as shown in Figure 1A, and constructed new nucleotide sequences codon-optimized for yeast.

For CR6261, the somatic and reconstructed germline heavy chain amino acid sequences were published in Lingwood et al., 2012. We used these sequences, similarly constructing codon-optimized nucleotide sequences for expression in yeast. The original somatic nucleotide sequence is also available (GenBank HI919029.1).

We note that all antibody libraries and clonal strains were constructed using somatic forms of the light chain, as these antibodies were isolated by combinatorial phage display (Throsby et al., 2008; Dreyfus et al., 2012), and so it is not possible to infer the naturally paired germline light chain sequence. Additionally, the CR9114 and CR6261 light chains were previously determined not to impact binding (Lingwood et al., 2012; Dreyfus et al., 2012; Ekiert et al., 2009). The somatic light chain sequence for CR9114 was obtained from Dreyfus et al., 2012 (GenBank JX213640.1), and that for CR6261 was obtained from Throsby et al., 2008 (GenBank HI919031.1).

\section{Mutation selection}

CR9114 contains a total of 18 amino acid substitutions between the somatic variant and the reconstructed germline sequence. However, a library of $2^{18}=262,144$ variants would be costly and timeconsuming to produce and assay via our methods. We therefore identified two mutations that were distant from antigen contacts in the crystal structure: A25S and E51D (Dreyfus et al., 2012). We measured binding affinities for somatic sequences with and without these two mutations, and found that these variants had comparable affinities for both $\mathrm{H} 1$ and $\mathrm{H} 3$ (Figure 1-figure supplement 7). Although these mutations may have some small impact on binding, especially in combination with others, excluding them allowed for a simpler cloning strategy and a more manageable library size.

Similar to the CR9114 library design, we reduced the number of mutations present in the CR6261 library by excluding three mutations that were distant from antigen contacts in the crystal structure: 6QE, L50P, and V101M (Ekiert et al., 2009). We validated the marginal contribution of these mutations to binding by measuring the binding affinities for the somatic sequence with and without these mutations reverted to the respective germline residue (Figure 1-figure supplement 7). 
Yeast display plasmid and strains

To generate clonal yeast display strains and libraries for CR9114, we cloned scFv constructs ( $V_{L}$-Ser (Gly $\left.{ }_{4} \mathrm{Ser}\right)_{5}-\mathrm{V}_{\mathrm{H}}-\mathrm{Myc}$ ) into the pCT302 plasmid (Midelfort et al., 2004) (kind gift from Dane Wittrup; Addgene, Watertown, MA, \#41845). For the clonal CR9114 somatic and germline strains, gene blocks corresponding to the somatic or inferred germline sequences were cloned into pCT302 by Gibson Assembly (Gibson et al., 2009) (plasmid maps in Supplementary files 4-5). For producing the plasmid backbone required for Golden Gate library generation (described below), we removed an existing Bsa-I site from the pCT302 plasmid by site-directed mutagenesis (Agilent, Santa Clara, $C A, \# 200521)$ and replaced the $V_{H}$ domain with the $c c d B$ gene. To generate clonal yeast strains, Gibson Assembly products were transformed into electrocompetent DH10B E. coli cells, and the resulting plasmids were mini-prepped and Sanger sequenced. Following sequence confirmation, plasmids were transformed into EBY100 yeast cells (ATCC \#MYA-4941) as described in the high-efficiency yeast transformation protocol (Gietz and Schiestl, 2007). Transformants were plated on SDCAAagar (1.71 g/L YNB without amino acids and ammonium sulfate [Sigma-Aldrich \#Y1251], $5 \mathrm{~g} / \mathrm{L}$ ammonium sulfate [Sigma-Aldrich \#A4418], 2\% dextrose [VWR \#90000-904], $5 \mathrm{~g} / \mathrm{L}$ Bacto casamino acids [VWR \#223050], $100 \mathrm{~g} / \mathrm{L}$ ampicillin [VWR \#V0339], 2\% Difco Noble Agar [VWR \#90000-774]) and incubated at $30^{\circ} \mathrm{C}$ for $48 \mathrm{hr}$, single colonies were restruck on SDCAA-agar and again incubated at $30^{\circ} \mathrm{C}$ for $48 \mathrm{hr}$, and the resulting clonal yeast strains were verified to have the construct of interest by colony PCR. Construction of the yeast libraries is described below. All yeast strains were grown to saturation in SDCAA at $30^{\circ} \mathrm{C}$, supplemented with $5 \%$ glycerol, and stored at $-80^{\circ} \mathrm{C}$.

CR6261 clonal yeast display strains and libraries were generated in an identical manner to that of CR9114, except where noted below (see Supplementary files 6-7 for plasmid maps corresponding to the germline and somatic sequences).

\section{Golden gate assembly}

For CR9114, due to the number of mutations required and their positions along the heavy chain coding sequence, we designed a library cloning strategy using Golden Gate combinatorial assembly (Engler et al., 2008). We divided the heavy chain coding region into five roughly equal fragments, ranging from 79 to $85 \mathrm{bp}$ and each containing between 1 and 5 mutations. We added Bsal sites and additional overhangs to both ends of each fragment sequence, with cut sites carefully chosen so that the five fragments will assemble uniquely in their proper order within the plasmid backbone. For each fragment with $n$ mutations, we then ordered $2^{n}$ individual DNA duplexes with each possible combination of mutations (ranging from 2 to 32 versions for each fragment, a total of 66 fragments) from IDT (Coralville, IA) (see Supplementary file 2). By pooling the versions of each fragment in equal volumes, then pooling the five fragment pools in equimolar ratios, we obtained a randomized fragment mix containing all $2^{16}$ sequences present at approximately equal frequencies.

In addition to the fragment mix, we prepared the plasmid backbone for the Golden Gate reaction. We created a version of the yeast display plasmid with the counter-selection marker $\operatorname{ccd} B$ in place of the heavy chain sequence, with flanking Bsal sites (see above). We performed Golden Gate cloning using Bsal-HFv2 (NEB, Ipswich, MA, \#R3733) following the manufacturer recommended protocol, with a 5:1 molar ratio of the fragment insert pool to plasmid backbone.

We transformed the assembly mix into electrocompetent E. coli (DH10B) via electroporation in 10 x $50 \mu \mathrm{L}$ cell aliquots. We recovered each transformation in $5 \mathrm{~mL} \mathrm{SOC} \mathrm{(2 \%} \mathrm{tryptone,} 0.5 \%$ yeast extract, $10 \mathrm{mM} \mathrm{NaCl}, 2.5 \mathrm{mM} \mathrm{KCl}, 10 \mathrm{mM} \mathrm{MgCl}, 10 \mathrm{mM} \mathrm{MgSO}_{4}, 20 \mathrm{mM}$ glucose) at $37^{\circ} \mathrm{C}$ for $1 \mathrm{hr}$, and then transferred each to $100 \mathrm{~mL}$ of molten LB (1\% tryptone, $0.5 \%$ yeast extract, $1 \% \mathrm{NaCl}) \mathrm{con}-$ taining $0.3 \%$ SeaPrep agarose (VWR, Radnor, PA \#12001-922) spread into a thin layer in a $1 \mathrm{~L}$ baffled flask (about $1 \mathrm{~cm}$ deep). The mixture was allowed to set on ice for an hour, after which it was kept for $18 \mathrm{hr}$ at $37 \mathrm{C}$ to allow for dispersed growth of colonies in $3 \mathrm{D}$. We observed $\sim 3 \times 10^{5}$ colonies per aliquot, for a total of $\sim 3$ million transformants. After mixing the flasks by shaking for $1 \mathrm{hr}$, we pelleted the cells and prepared plasmid by standard midiprep (Zymo Research, Irvine, CA, D4201), from which we obtained $>120 \mu \mathrm{g}$ of purified plasmid.

For CR6261, we designed a library cloning strategy also using Golden Gate combinatorial assembly, but with fragments created by PCR instead of purchased. We divided the heavy chain coding region into three roughly equal fragments, each containing between 2 and 5 mutations. We designed these fragments such that the mutations they contain are close to the $3^{\prime}$ or $5^{\prime}$ ends and 
can thus be easily incorporated by PCR. PCR primers included mutations, Bsal sites, and unique overhangs chosen so that the three fragments would assemble uniquely in their proper order within the plasmid backbone. For each version of the three fragments, we generated dsDNA by PCR (52 PCR reactions in total; see Supplementary file 3 for primer sequences). By pooling all versions of each fragment in equal volumes, then pooling the three fragment pools in equimolar ratios, we obtain a randomized fragment mix that, when ligated in the Golden Gate reaction, produces all of the $2^{11}$ sequences present at approximately equal frequencies.

In addition to the fragment mix, we prepared the plasmid backbone for the Golden Gate reaction. We created a version of the yeast display plasmid with the counter-selection marker $\operatorname{ccd} B$ in place of the three-fragment sequence, with flanking Bsal sites. We performed Golden Gate cloning using Bsal-HFv2 (NEB \#R3733) following the manufacturer recommended protocol, with a 7:1 molar ratio of fragment inserts to plasmid backbone.

The transformation of the CR6261 library into E. coli was conducted in a similar fashion to that of CR9114, except that $8 \times 50 \mu \mathrm{L}$ cell aliquots were transformed, and 600,000 colonies were pooled for plasmid midiprep.

\section{Yeast library production}

We then transformed the CR9114 plasmid library into EBY100 cells by standard high-efficiency protocols (Gietz and Schiestl, 2007). We recovered transformants in molten SDCAA (1.71 g/L YNB without amino acids and ammonium sulfate (Sigma-Aldrich \#Y1251), $5 \mathrm{~g} / \mathrm{L}$ ammonium sulfate (Sigma-Aldrich, St. Louis, MO, \#A4418), 2\% dextrose (VWR \#90000-904), 5 g/L Bacto casamino acids (VWR \#223050), $100 \mathrm{~g} / \mathrm{L}$ ampicillin (VWR \# V0339)) containing 0.35\% SeaPrep agarose (VWR \#12001-922) spread into a thin layer (about $1 \mathrm{~cm}$ deep). The mixture was allowed to set on ice for an hour, after which it was kept for $48 \mathrm{hr}$ at $30^{\circ} \mathrm{C}$ to allow for dispersed growth of colonies in 3D. From five such flasks, we obtained $\sim 700,000$ colonies ( $>10$ times the library diversity). After mixing the flasks thoroughly by shaking for $1 \mathrm{hr}$, we grew cells in $5 \mathrm{~mL}$ tubes of liquid SDCAA for five generations and froze the saturated culture in $1 \mathrm{~mL}$ aliquots with $5 \%$ glycerol.

The CR6261 yeast library was generated in a manner identical to that of CR9114, except that $\sim 60,000$ colonies were pooled due to the smaller library size.

\section{Isogenic strain production}

In addition to the full library, for both CR9114 and CR6261 we assayed a small number of variants by low-throughput flow cytometry for Tite-Seq validation. Any individual variant in the library can be produced in the same manner as described above: we simply selected the DNA duplex fragments corresponding to each desired variant and set up an individual Golden Gate reaction. The resulting assembled plasmid was transformed into E. coli, mini-prepped, and transformed into EBY100 in the same manner as described above. We verified the sequence identity of each variant by Sanger sequencing the entire scFv sequence.

We also constructed isogenic strains for validation experiments with genotypes that are not present in the full library. For CR9114, to test the impact of excluding mutations A24S and E46D, we constructed a strain containing the remaining 16 somatic mutations by cloning a gene block of the corresponding $\mathrm{V}_{\mathrm{H}}$ sequence into the germline CR9114 pCT302 plasmid via Gibson Assembly (Figure 1-figure supplement 7). For CR6261, we similarly constructed a strain with the Q6E, L50P, and V101M mutations reverted.

\section{Antigen production}

Choice of HA antigens

CR9114 was isolated from pooled PBMC from three donors who had received the trivalent 2006 influenza vaccine (Throsby et al., 2008; Dreyfus et al., 2012), which contained A/New Caledonia/ 20/1999 (H1N1), A/Wisconsin/67/2005 (H3N2), and B/Malaysia/2506/2004 (Victoria lineage) (Ekiert et al., 2011). CR6261 was isolated from pooled PBMC from the same three donors, plus an additional seven donors who did not receive the vaccine (Throsby et alo, 2008). Because PBMC were isolated only 7 days after vaccination, although it is possible that CR6261 and CR9114 matured in response to these specific antigens, it is more likely that the vaccine elicited memory recall of these antibodies (Victora and Nussenzweig, 2012). Here, we chose to measure binding affinities to 
diverse antigens spanning the range of breadth for both CR9114 and CR6261. CR9114 neutralizes strains across influenza $A$ (groups 1 and 2) and influenza $B$, so we measured affinities to one strain from each of these groups, and selected vaccine-like strains: A/New Caledonia/20/1999 (H1N1), A/ Wisconsin/67/2005 (H3N2), and B/Ohio/1/2005 (Victoria lineage). CR6261 neutralizes strains across influenza A group 1, thus we measured affinities to two strains from distinct subtypes within group 1: A/New Caledonia/20/1999 (H1N1) and A/Hong Kong/1073/1999 (H9N2). We note that CR9114 indeed binds A/Hong Kong/1073/1999 (H9N2) (Dreyfus et al., 2012), but CR9114 variant affinities for this strain were not measured here, as we prioritized measurements to antigens that span the breadth of each antibody.

\section{HA cloning, expression, and purification}

Trimeric hemagglutinin (HA) antigen was produced as previously described (Ekiert et al., 2011; Dreyfus et al., 2012; Margine et al., 2013). Briefly, the HA ectodomain (Influenza A: residues 11329 of HA1 and 1-176 of HA2 (H3 numbering); Influenza B: residues 1-523) of Influenza A/New Caledonia/1999 H1, Influenza A/Hong Kong/1999 H9, Influenza A/Wisconsin/2005 H3, and Influenza B/ Ohio/2005, with N-terminal gp67 signal peptide and C-terminal biotinylation site (GGGLNDIFEA QKIEWHE), thrombin cleavage site, trimerization domain and $\mathrm{His}_{6}$ tag, were cloned into pFastbac (plasmid maps in Supplementary files 8-11). Recombinant bacmid was generated using the ThermoFisher Bac-to-Bac kit (ThermoFisher, Waltham, MA, \#10359016). Sf9 cells (ThermoFisher \#B82501, not authenticated but verified to be mycoplasma-negative) were then transfected (ThermoFisher \#A38915, not authenticated but verified to be mycoplasma-negative) with the resulting bacmids, and PO HA-baculovirus was harvested 7 days post-transfection by clarifying viral supernatant at $1000 \times \mathrm{g}$ for $10 \mathrm{~min}$. HA-baculovirus was then amplified twice by successively infecting 187 million Sf9 cells with $100 \mu \mathrm{L}$ of viral supernatant and incubating in a humidified incubator at $28^{\circ} \mathrm{C}$ for 12 days. To induce HA expression, 105 million High-Five cells (ThermoFisher \#B85502) were resuspended with $15 \mathrm{~mL}$ P2 HA-baculovirus, incubated for $20 \mathrm{~min}$ at room temperature, and then transferred to a $1 \mathrm{~L}$ non-baffled flask with $200 \mathrm{~mL}$ Corning Express-Five media (ThermoFisher \#10486025) supplemented with 18 mM L-glutamine (VWR \#45000-676). Expression cultures were incubated in a shaking incubator at $28 \mathrm{C}$ and $110 \mathrm{rpm}$ for $48 \mathrm{hr}$, after which HA-containing media was clarified by spinning first at $1000 \times \mathrm{g}$ for $5 \mathrm{~min}$ at $4^{\circ} \mathrm{C}$, and then by spinning the resulting supernatant again at $4000 \times \mathrm{g}$ for $20 \mathrm{~min}$ at $4 \mathrm{C}$. The clarified media was then dialyzed into PBS (VWR \#45000-448) by performing $4 \times 2 \mathrm{hr} 10$-fold buffer exchanges to remove metal chelators from culture media. Dialyzed media was then combined with $10 \mathrm{~mL}$ equilibrated NiNTA resin (ThermoFisher \#R90101), gently shaken for $3 \mathrm{hr}$ at $4^{\circ} \mathrm{C}$, and loaded onto a column. The resin was washed first with 15 column volumes Wash Buffer 1 (50 mM Tris pH 8 at 4C, $300 \mathrm{mM} \mathrm{KCl}, 10 \mathrm{mM}$ imidazole) and subsequently with 15 column volumes Wash Buffer 2 (50 mM Tris pH 8 at 4C, $300 \mathrm{mM} \mathrm{KCl}, 20 \mathrm{mM}$ imidazole). HA was eluted from the resin after $10 \mathrm{~min}$ incubation with Elution Buffer $\left(50 \mathrm{mM}\right.$ Tris $\mathrm{pH} 8$ at $4^{\circ} \mathrm{C}, 300 \mathrm{mM} \mathrm{KCl}, 250$ $\mathrm{mM}$ imidazole). HA was then buffer exchanged into PBS using $10 \mathrm{KDa}$ Amicon Ultra Centrifugal Filters (Millipore Sigma, Burlington, MA \#UFC901008) and concentrated to at least $1 \mathrm{mg} / \mathrm{mL}$ for downstream biotinylation.

\section{BirA expression and purification}

BirA was expressed and purified as previously described (Ekiert et alo, 2011). Briefly, pET21a-BirA expression plasmid (Howarth et al., 2005) (kind gift from Alice Ting; Addgene \#20857) was transformed into BL21 (DE3). Transformed BL21 cells were grown in $4 \mathrm{~L}$ baffled flasks with $1 \mathrm{~L}$ low-salt LB medium (5 g/L NaCl, $5 \mathrm{~g} / \mathrm{L}$ yeast extract (VWR \#90000-722), $10 \mathrm{~g} / \mathrm{L}$ tryptone (VWR \#90000-286)) at $37^{\circ} \mathrm{C}$ to an OD $(600 \mathrm{~nm})$ of $\sim 0.8$. The culture was then moved into cold water to bring it to $23^{\circ} \mathrm{C}$, IPTG was added to a final concentration of $1 \mathrm{mM}$, and the culture was incubated at 23C for $\sim 16 \mathrm{hr}$. The culture was then harvested by centrifugation (3000 x g, $10 \mathrm{~min})$, resuspended in $30 \mathrm{~mL}$ lysis buffer $(50 \mathrm{mM}$ Tris $\mathrm{pH} 8$ at 4C, $300 \mathrm{mM} \mathrm{KCl}, 10 \mathrm{mM}$ imidazole, EDTA-free protease inhibitor cocktail tablet (Millipore Sigma \#4693159001)), lysed by sonication (Branson Sonifier 450), and shaken at $4^{\circ} \mathrm{C}$ for $30 \mathrm{~min}$. Lysate was clarified by spinning at 25,000 x g for $1 \mathrm{hr}$, and then the supernatant was incubated with $5 \mathrm{~mL}$ NiNTA resin at $4^{\circ} \mathrm{C}$ for $3 \mathrm{hr}$ with gentle shaking. The resin was pelleted by spinning at $500 \times \mathrm{g}$ for $5 \mathrm{~min}$ and washed twice by gentle shaking with $35 \mathrm{~mL}$ lysis buffer at $4^{\circ} \mathrm{C}$ for $30 \mathrm{~min}$. Protein was eluted with $20 \mathrm{~mL}$ Elution Buffer $\left(50 \mathrm{mM}\right.$ Tris $\mathrm{pH} 8$ at $4^{\circ} \mathrm{C}, 300 \mathrm{mM} \mathrm{KCl}, 250 \mathrm{mM}$ 
imidazole), buffer exchanged into Storage Buffer $\left(50 \mathrm{mM}\right.$ Tris $\mathrm{pH} 7.5$ at $4^{\circ} \mathrm{C}, 200 \mathrm{mM} \mathrm{KCl}, 5 \%$ glycerol) using $10 \mathrm{KDa}$ Amicon Ultra Centrifugal Filters (Millipore Sigma \#UFC901008), flash frozen in liquid nitrogen, and stored in single-use aliquots at $-80^{\circ} \mathrm{C}$.

\section{Biotinylation and HA-biotin quality control}

Purified hemagglutinin was biotinylated as previously described (Fairhead and Howarth, 2015; Ekiert et al., 2011). Briefly, $100 \mu \mathrm{L} \mathrm{HA}\left(>1 \mathrm{mg} / \mathrm{mL}\right.$ ) was incubated with $0.5 \mu \mathrm{L} 1 \mathrm{M} \mathrm{MgCl}_{2}, 2 \mu \mathrm{L} 100$ $\mathrm{mM}$ ATP, $0.5 \mu \mathrm{L} 50 \mathrm{mM}$ biotin, and $2.5 \mu \mathrm{L} \operatorname{BirA}(10 \mathrm{mg} / \mathrm{mL})$. This was mixed by gentle pipetting and incubated at $30 \mathrm{C}$ with gentle rocking. After $1 \mathrm{hr}$ incubation, equivalent amounts of ATP, BirA, and biotin were added to the reaction, which was incubated for an additional hour at $30^{\circ} \mathrm{C}$. Following the $2 \mathrm{hr}$ incubation, the $100 \mu \mathrm{L}$ reaction was exchanged thrice into $15 \mathrm{~mL}$ PBS using a 50 KDa MWCO buffer exchange column (Millipore Sigma \#UFC905008). The degree of biotinylation was then assessed by a streptavidin gel-shift assay, as previously described (Fairhead and Howarth, 2015). Briefly, 10-fold molar excess streptavidin (Millipore Sigma \#189730) was added to $4 \mu \mathrm{g}$ biotinylated $\mathrm{HA}$ and incubated at room temperature for $5 \mathrm{~min}$ prior to running on SDS-PAGE. Gels were transferred to nitrocellulose membranes and probed with mouse anti-His monoclonal antibodies (ThermoFisher \#R930-25) and Goat-anti-mouse secondary antibodies (LiCor, Lincoln, NE, Cat\#925-32210). HA was verified to be $>80 \%$ biotinylated by densitometry.

\section{Tite-Seq assays}

Tite-Seq was performed essentially as previously described (Adams et al., 2016), with some modifications as detailed below. For each antibody-antigen pair, three replicate Tite-Seq assays were performed on different days.

\section{Induction of antibody expression}

On day 1, yeast scFv libraries, as well as germline and somatic clonal strains, were thawed by inoculating $5 \mathrm{~mL}$ SDCAA (1.71 $\mathrm{g} / \mathrm{L}$ YNB without amino acids and ammonium sulfate (Sigma-Aldrich \#Y1251), $5 \mathrm{~g} / \mathrm{L}$ ammonium sulfate (Sigma-Aldrich \#A4418), 2\% dextrose (VWR \#90000-904), $5 \mathrm{~g} / \mathrm{L}$ Bacto casamino acids (VWR \#223050), $100 \mathrm{~g} / \mathrm{L}$ ampicillin (VWR \# V0339)) with $150 \mu \mathrm{L}$ glycerol stock (saturated culture with $5 \%$ glycerol) and rotated at $30^{\circ} \mathrm{C}$ for $20 \mathrm{hr}$. On day 2, yeast cultures were back-diluted to OD600 $=0.2 \mathrm{in} 5 \mathrm{~mL}$ SDCAA and rotated at $30^{\circ} \mathrm{C}$ for approximately $4 \mathrm{hr}$, or until reaching log phase $(O D 600=0.4-0.8) .1 .5 \mathrm{~mL}$ log-phase cells were then pelleted, resuspended in 4 $\mathrm{mL}$ SGDCAA (1.71 g/L YNB without amino acids and ammonium sulfate (Sigma-Aldrich \#Y1251), 5 $\mathrm{g} / \mathrm{L}$ ammonium sulfate (Sigma-Aldrich \#A4418), 0.2\% dextrose (VWR \#90000-904), 1.8\% galactose (Sigma-Aldrich \#G0625), $5 \mathrm{~g} / \mathrm{L}$ Bacto casamino acids (VWR \#223050), $100 \mathrm{~g} / \mathrm{L}$ ampicillin (VWR \#V0339)), and rotated at room temperature for 20-22 hr.

Primary antigen labeling

On day 3, 20-22 hr post-induction, yeast cultures were pelleted, washed twice with $0.1 \%$ PBSA (VWR \#45001-130; GoldBio, St. Louis, MO, \#A-420-50), and resuspended to an OD600 of 1. A total of $700 \mu \mathrm{L}$ of OD1 yeast cells were labeled with biotinylated HA at each of eleven antigen concentrations (half-log increments spanning $1 \mathrm{pM}-100 \mathrm{nM}$ for $\mathrm{H} 1$ and $\mathrm{H} 9$, and $10 \mathrm{pM}-1 \mu \mathrm{M}$ for $\mathrm{H} 3$ and influenza $B$, as well as no $\mathrm{HA}$ ), with volumes adjusted such that the number of antigen molecules was in 10 -fold excess of antibody molecules (assuming 50,000 scFv/cell). Yeast-HA mixtures were rocked at $4^{\circ} \mathrm{C}$ for $24 \mathrm{hr}$.

\section{Secondary labeling}

On day 4, yeast-HA complexes were pelleted by spinning at $3000 \times \mathrm{g}$ for $10 \mathrm{~min}$ at $4^{\circ} \mathrm{C}$, washed twice with 5\% PBSA + 2 mM EDTA, and simultaneously labeled with Streptavidin-RPE (1:100, Thermo Fisher \#S866) and anti-cMyc-FITC (1:50, Miltenyi Biotec, Somerville, MA, \#130-116-485) at $4^{\circ}$ $\mathrm{C}$ for $45 \mathrm{~min}$. Following secondary labeling, yeast were washed twice with 5\% PBSA + 2 mM EDTA, and left on ice in the dark until sorting. 
Sorting and recovery

Yeast were sorted on a BD FACS Aria Illu, equipped with $405 \mathrm{~nm}, 440 \mathrm{~nm}, 488 \mathrm{~nm}, 561 \mathrm{~nm}$, and 635 $\mathrm{nm}$ lasers, and an 85 micron fixed nozzle. Prior to sorting, single-color controls were used to compensate for the minimal FITC overlap with PE. Single cells were gated by FSC vs SSC, and then this population was sorted either by expression (FITC) or by expression and binding (PE). For all sorts, at least ten-fold excess of the library diversity was sorted ( 1.6 million cells for CR9114; $\sim 500,000$ cells for CR6261). For the expression sorts, singlets were sorted into eight equivalent FITC log-spaced gates. For the binding sorts, FITC-positive cells were sorted into 4 PE bins (the PE-negative population comprised bin 1, and the PE-positive population was split into three equivalent log-spaced bins 2-4; see Figure 1-figure supplement 6). Polypropylene collection tubes were coated and filled with $1 \mathrm{~mL}$ YPD supplemented with 1\% BSA and placed on ice until recovery. Sorted cells were pelleted by spinning at $3000 \times \mathrm{g}$ for $10 \mathrm{~min}$, and supernatant was removed by pipette to avoid disturbing the pellets. Pellets were then resuspended in $4 \mathrm{~mL}$ SDCAA, a small amount was plated on SDCAA-agar to quantify recovery efficiency, and cultures were rocked at $30^{\circ} \mathrm{C}$ until reaching late-log phase $(\mathrm{OD} 600=0.6-1.2)$.

\section{Sequencing library preparation}

A total of $1.5 \mathrm{~mL}$ of late-log yeast cultures were pelleted and scFv plasmid was extracted using Zymo Yeast Plasmid Miniprep II (Zymo Research \# D2004), per the manufacturer's instructions, and eluted in $10 \mu \mathrm{L}$ elution buffer. Heavy-chain amplicon sequencing libraries were prepared by a twostep PCR as previously described (Nguyen Ba et alo, 2019). In the first PCR, unique molecular identifiers (UMI), inline indices, and partial Illumina adapters were appended to the heavy chain through 3-5 amplification cycles to minimize PCR amplification bias. In the second PCR, the remainder of the Illumina adapter and sample-specific Illumina i5 and i7 indices were appended through 35 amplification cycles (see Supplementary file 1 for primer sequences). The first PCR used $5 \mu \mathrm{L}$ plasmid DNA as template in a $25 \mu \mathrm{L}$ reaction volume, with $\mathrm{Q} 5$ polymerase according to the manufactuer's instructions (NEB \# M0491L), and was incubated in a thermocycler with the following program: $1.60 \mathrm{~s}$ at $98^{\circ} \mathrm{C}, 2.10 \mathrm{~s}$ at $98^{\circ} \mathrm{C}, 3.30 \mathrm{~s}$ at $66^{\circ} \mathrm{C}, 4.30 \mathrm{~s}$ at $72^{\circ} \mathrm{C}, 5$. GOTO 2, 2-4x, $6.60 \mathrm{~s}$ at $72^{\circ} \mathrm{C}$. PCR products were then combined with carrier RNA and purified by 1.1X Aline beads (Aline Biosciences \#C-10035), and eluted in $35 \mu \mathrm{L}$ elution buffer. $33 \mu \mathrm{L}$ of the elution was used as input for the second PCR, in a total volume of $50 \mu \mathrm{L}$ using Kapa polymerase (Kapa Biosystems \#KK2502) according to the manufacturer's instructions, and incubated in a thermocycler with the following program: $1.30 \mathrm{~s}$ at $98^{\circ} \mathrm{C}, 2$. $20 \mathrm{~s}$ at $98^{\circ} \mathrm{C}, 3.30 \mathrm{~s}$ at $62^{\circ} \mathrm{C}, 4.30 \mathrm{~s}$ at $72^{\circ} \mathrm{C}, 5$. GOTO $2,34 \mathrm{x}, 6.300 \mathrm{~s}$ at $72^{\circ} \mathrm{C}$. The resulting sequencing libraries were purified by $0.85 \mathrm{X}$ Aline beads, amplicon size was verified to be $\sim 500$ bp by running on a $1 \%$ agarose gel, and amplicon concentration was quantified by a fluorescent DNA-binding dye (Biotium, Fremont, CA, \#31068, per manufacturer's instructions). Amplicons were then pooled for each gate according to the number of sorted cells to ensure even sequencing coverage. The pool was further size-selected by a two-sided Aline bead cleanup (0.55-0.85X), and the final pool size was verified by Tapestation $5000 \mathrm{HS}$ and $1000 \mathrm{HS}$. Final sequencing library concentration was determined by Qubit fluorometer and sequenced on an Illumina NovaSeq S2 or Miseq v3 (2x150) with 5\% PhiX.

\section{Sequencing data processing}

We first processed our raw sequencing reads to identify and extract the indexes and mutational sites, discarding priming regions and the constant regions between mutations. To do so, we developed custom Python scripts using the approximate regular expression library regex (Barnett, 2013), which allowed us to handle complications in sequence parsing that arise from the irregular lengths of the indices and from sequencing errors. We accept sequences that match the entire read (with no restrictions on bases at mutational sites) within the following mismatch tolerances: two mismatches in the multiplexing index, two mismatches in the priming site, and 15 substitution mismatches within the 170 bases of constant antibody sequence.

We then examine the mutational sites to call germline or somatic alleles, producing binary genotypes (' 0 ' for germline or ' 1 ' for somatic at each position). We require the exact germline or somatic sequence at every site: if there are any substitution errors in any of the mutation sites, the entire read is rejected. While it is possible to perform error correction based on Hamming distance to 
rescue reads with a few substitution errors, we find that on average only $<8 \%$ of reads per sample contain any errors, and so we adopt the conservative approach of requiring perfect matching.

We next discarded sequencing reads with any mismatched indices (four total indices from the two PCR reactions), as well as reads with duplicate UMI sequences. Counts for each genotype were then tabulated, producing the final counts used for binding affinity inference (see below). On average, across all antigens and replicates, we obtain a mean coverage of $\sim 350$ for CR9114 and $\sim 950$ for CR6261, and a median coverage of $\sim 250$ for CR9114 and $\sim 900$ for CR6261.

\section{Isogenic validation}

Induction of scFv surface display, primary labeling, and secondary labeling of isogenic strains were performed identically to the Tite-Seq assay, except yeast cell and antigen volumes were scaled down by a factor of 10. Yeast cell FITC (scFv expression) and R-PE (HA binding) fluorescence intensity was assayed on a BD LSR Fortessa equipped with four lasers (440, 488, 561, and $633 \mathrm{~nm})$. The equilibrium binding affinities $\left(K_{D}\right)$ for each variant are inferred by fitting the log of a Hill function to the mean log R-PE fluorescence of scFv-expressing (FITC+) singlet yeast cells:

$$
\text { mean log fluorescence }=\log _{10}\left(A_{s} \frac{c}{c+K_{D, s}}+B_{s}\right),
$$

where $c$ is the antigen concentration in molar units, $A_{s}$ is the increase in fluorescence due to saturation with antigen, $B_{s}$ is the background fluorescence, and $K_{D, s}$ is the equilibrium binding affinity. All isogenic measurements were performed in two to three biological replicates; see Figure 1-source data 3 for isogenic $-\log _{10} K_{D}$.

\section{Tite-Seq binding affinity inference}

\section{Mean-bin approach}

To infer binding affinities using a simple mean-bin approach (Peterman and Levine, 2016), we incorporate sequencing data (the unique read counts of each genotype sequence $s$ in bin $b$ at concentration $c, R_{b, s, c}$ ) with flow cytometry data (the mean and standard deviation of $\log _{10}$-fluorescence of sorted cells in each bin $b$ at concentration $c, F_{b, c}$ and $\sigma_{F_{b, c}}$ respectively, and cell counts for each bin $b$ at each concentration $c, C_{b, c}$ ).

The mean log-fluorescence of each genotype sequence at each of the 12 antigen concentrations is calculated as:

$$
\bar{F}_{s, c}=\sum_{b} F_{b, c} p_{b, s \mid c}
$$

where $p_{b, s \mid c}$ is the probability a cell with sequence $s$ would be sorted into bin $\mathrm{b}$ at concentration $\mathrm{c}$ is estimated from the sequencing read counts as:

$$
p_{b, s \mid c}=\frac{\frac{R_{b, s, c}}{\sum_{s^{\prime}} R_{b, s^{\prime}, c}} \cdot C_{b, c}}{\sum_{b^{\prime}}\left(\frac{R_{b^{\prime}, s, c}}{\sum_{s^{\prime}} R_{b^{\prime}, s^{\prime}, c}} \cdot C_{b^{\prime}, c}\right)},
$$

in other words, the fraction of total reads in the bin corresponding to sequence $s$, scaled by the number of sorted cells in that bin, normalized over the four bins for each concentration.

The uncertainty in the mean bin inference was propagated as:

$$
\delta \bar{F}_{s, c}=\sqrt{\sum_{b}\left(\delta F_{b, c}^{2} p_{b, s \mid c}^{2}+F_{b, c}^{2} \delta p_{b, s \mid c}^{2}\right)} .
$$

Here, $\delta F_{b, c}$ represents the spread in log-fluorescence values of cells sorted into the same bin $b$. While we could estimate this value using the bin width, in practice we find that the distribution of cell log-fluorescence values in a bin is far from uniform across the bin width. The distribution is often not normal either, but we find that approximating $\delta F_{b, c} \approx \sigma_{F_{b, c}}$ or the standard deviation in $\log _{10}$-fluorescence of cells sorted into bin $b$ at concentration $c$, adequately captures the typical variation. The 
error in $p_{b, s \mid c}$ arises largely from the sampling process of sequencing, which can be approximated as a Poisson process when read counts are relatively high. This gives

$$
\delta p_{b, s \mid c}=\frac{p_{b, s \mid c}}{\sqrt{R_{b, s, c}}}
$$

Thus, $\delta \bar{F}_{s, c}$ can be written as

$$
\delta \bar{F}_{s, c}=\sqrt{\sum_{b}\left(\sigma_{F_{b, c}}^{2} p_{b, s \mid c}^{2}+F_{b, c}^{2} \frac{p_{b, s \mid c}^{2}}{R_{b, s, c}}\right)} .
$$

The equilibrium binding affinities $\left(K_{D}\right)$ for each variant are inferred by fitting the logarithm of a Hill function to the resulting mean $\log _{10}$-fluorescence across the twelve antigen concentrations:

$$
\bar{F}_{s, c}=\log _{10}\left(A_{s} \frac{c}{c+K_{D, s}}+B_{s}\right)
$$

where $c$ is the antigen concentration in molar units, $A_{s}$ is the increase in fluorescence due to saturation with antigen, $B_{s}$ is the background fluorescence, and $K_{D, s}$ is the binding affinity. Fitting was performed with the curve_fit function of the Python package scipy.optimize. Reasonable bounds on the values of $A\left(10^{3}-10^{5}\right), B\left(10^{0}-10^{3}\right)$ and $K_{D}\left(10^{-14}-10^{-5}\right)$ were imposed. Sequences leading to a failed optimization were deemed 'non-binding'.

Inferred $K_{D}$ outside of the titration boundaries were then pinned to the boundaries $\left(10^{-12}\right.$ and $10^{-7}$ for $\mathrm{H} 1$ and $\mathrm{H} 9 ; 10^{-11}$ and $10^{-6}$ for $\mathrm{H} 3$ and FluB). Inferred $K_{D}$ with high error (standard deviation of $\left.\log _{10} K_{D}>1.0\right)$ or resulting from a poor fit $\left(r^{2}<0.8\right)$ were removed from the data set prior to averaging $-\log _{10} K_{D}$ values across biological replicates.

We also explored an alternative maximum-likelihood framework for inferring binding affinities (see Appendix 1), but found it to be less accurate than the mean-bin approach when compared to isogenic flow cytometry measurements. Thus, we restricted our analysis to the simpler and more robust mean-bin inference presented here.

\section{Force-directed layouts}

To represent the high-dimensional binding affinity landscape in two dimensions, we use a forcedirected graph layout approach. Each sequence in the antibody library is a node, connected by edges to its single-mutation neighbors (sequences that can be reached by one additional somatic mutation). An edge between two sequences $s$ and $t$ is given the weight

$$
w_{s, t}=\frac{1}{0.01+\left|\log _{10}\left(K_{D, s}^{\mathrm{ag}}\right)-\log _{10}\left(K_{D, t}^{\mathrm{ag}}\right)\right|} \text {, }
$$

where $K_{D}^{\mathrm{ag}}$ represent binding affinities to a particular antigen, ag. In the layouts shown in the main text, we use binding affinities to $\mathrm{H} 1$ for both CR6261 and CR9114. In force-directed layouts, edge weights correspond to the effective spring constant that tends to pull nodes closer together. Thus, a mutation from sequence $s$ to $t$ that has little impact on binding will cause that edge weight to be large, and the nodes will be pulled strongly together. A mutation from sequence $s$ to $t$ that causes a large difference in binding affinity (positive or negative) to the antigen will reduce the edge weight, moving those nodes further apart. After assigning all edge weights, we use the layout function layout_drl from the Python package iGraph, with default settings, to obtain the layout coordinates for each variant.

\section{Expression data}

As noted above, antibody libraries were sorted into eight bins along the FITC-A fluorescence axis (where FITC-A fluorescence is proportional to expression), each comprising $12.5 \%$ of the total singlet population (Figure 1-figure supplement 6). The mean expression log-fluorescence was computed for each variant using the corresponding variant counts and fluorescence data, as described above for the mean-bin $K_{D}$ inference. These expression values were then averaged across all biological 
replicates for each antibody (nine replicates for CR9114, six replicates for CR6261), and correlation between biological replicates, as well as with $-\log _{10} K_{D}$ values, are illustrated in Figure 1-figure supplement 5. For the isogenic flow cytometry measurements, variant expression was computed as the mean log FITC-A fluorescence.

\section{Epistasis analysis}

\section{Linear interaction models}

To infer specific mutational effects, we begin with simple linear models where the effects of mutations (and mutation combinations) add to produce phenotypes. Our log-transformed phenotypes for each variant $s, y_{s}=-\log _{10}\left(K_{D, s}\right)$, are proportional to free-energy changes, and thus a natural null expectation is that they combine additively (Wells, 1990; Olson et al., 2014) (although we also consider nonadditive epistatic interactions between individual loci here, and analyze the effects of an overall nonlinear transformation of this data in Appendix 2). Our additive-only model is

$$
y_{s}=\beta_{0}+\sum_{i=1}^{L} \beta_{i} x_{i, s}+\varepsilon
$$

where $L$ is the number of mutations for a given antibody, $\beta_{0}$ is an intercept term, $\beta_{i}$ is the effect of the mutation at site $i, x_{i, s}$ is the genotype of variant $s$ at site $i$, and $\varepsilon$ represents independently and identically distributed errors. Our general linear interaction models are

$$
y_{s}=\beta_{0}+\sum_{i} \beta_{i} x_{i, s}+\sum_{i<j}^{L} \beta_{i j} x_{i, s} x_{j, s}+\sum_{i<j<k}^{L} \beta_{i j k} x_{i, s} x_{j, s} x_{k, s}+\ldots+\varepsilon
$$

where $\beta_{i j}$ represent second-order interaction coefficients between distinct sites $i$ and $j, \beta_{i j k}$ represent third-order interaction coefficients, and so on up to the desired maximum order of interaction.

There are multiple alternative coding systems for the binary genotypes $x_{i, s}$ that affect the values of inferred effects $\beta$ as well as their interpretation. Two common choices are (1) $x_{i, s} \in\{0,1\}$, often called 'biochemical' or 'local' epistasis, and (2) $x_{i, s} \in\{-1,1\}$, often called 'statistical' or 'ensemble' epistasis (Poelwijk et al., 2016). These frameworks are equivalent and related by a simple linear transformation, but the values of the coefficients vary between frameworks and have different interpretations. For ease of interpretation, in the Main Text and Figures we always show results obtained from inference in the biochemical epistasis framework. In Appendix 2, we discuss the differences between these two frameworks, and present results from inference in the statistical epistasis framework.

For an antibody with $L$ mutations, there are $L$ possible orders of interactions, with a total of $2^{L}$ epistatic coefficients $\beta$. From a measurement of $y$ for all $2^{L}$ possible sequences, there is a simple linear transformation to calculate the resulting $2^{L}$ parameters (Poelwijk et al., 2016). This is a simple and fast approach to the calculation of epistasis that is widely used (Sailer and Harms, 2017b; Poelwijk et al., 2019), and we explore this approach in Appendix 2. However, we may instead wish to restrict our model to a lower order and examine whether it can explain the data with far fewer than $2^{L}$ parameters, as a conservative approach to detecting high-order epistasis.

Specifically, we truncate the model above at a maximum order $n$ and then fit and evaluate the resulting model. We begin with $n=1$ and continue to increase $n$ until the optimal model has been identified. There are multiple strategies for selecting between models with different numbers of parameters, such as AIC and BIC; here we take a cross-validation approach. For each fold, we hold out $10 \%$ of the dataset, train models at each maximum order on the remaining $90 \%$, and evaluate the prediction performance $\left(R^{2}\right)$ of the model on the held-out test set. After averaging the performances across all 10 folds for each truncated model, we choose the order that maximizes the test set performance as the optimal maximal order of interaction. We then re-train the model truncated at this order on the full dataset to obtain the final coefficients. We find that the optimal model identified by cross-validation for each antibody-antigen pair satisfies $p<N$ by $\sim 1$ order of magnitude, where $p$ is the total number of model coefficients and $N$ the number of data points with measurable binding affinity. This gives confidence that our parameter estimates are well constrained by the data, even in the absence of other regularization (such as Lasso or Ridge regularization approaches). 
To train a model of given order on a set of sequences, we use ordinary least squares (OLS) regression with the Python package statsmodels. From this, we obtain the coefficient values $\beta$ with their standard errors and p-values. To define significance of coefficients, we use a $p$-value cutoff of 0.05 with Bonferroni correction by the total number of model parameters. Coefficients, standard errors, p-values, and Bonferroni-corrected $95 \%$ confidence intervals are reported in Figure 1source data 1 and 2 . We also predict phenotypes $\hat{y}$ for each sequence from the coefficients and use these values in Figure 5A, B.

For CR9114 binding to influenza B, the number of sequences used for inference is far fewer than other antibody-antigen pairs $(N=256)$, due to the large number of required mutations. We therefore use a fivefold rather than 10 -fold split to reduce the test set noise. Nevertheless, the cross-validation procedure identifies a first-order (additive) model as optimal, due to the smaller sample size.

\section{Structural analysis of epistatic coefficients}

To examine the structural context of linear and pairwise coefficients, we performed three simple analyses. (1) First, we used ChimeraX (Pettersen et al., 2021) to calculate the buried surface area between HA and each mutated residue in CR9114 and CR6261, using the measure buriedarea function and the default probeRadius of 1.4 angstroms to approximate a water molecule. We plot this 'contact surface area' vs the linear effect of the corresponding mutation on HA binding (Figure 2C; Figure 2-figure supplement 1A). (2) We used PyMol (Schrodinger, LLC, 2015) to count the number of HA residues within six angstroms of each antibody mutation site. Six angstroms was chosen as an upper limit to capture potential antibody-antigen interactions (Bondi, 1964; Baker and Hubbard, 1984; Israelachvili and Pashley, 1982; Ekiert et al., 2009; Dreyfus et al., 2012), although we note that this analysis is robust to other distance thresholds. (3) We also used PyMol to measure the distances between $\alpha$-carbons for all mutation pairs, and plotted these distances against the corresponding pairwise epistatic terms (Figure 2F; Figure 2-figure supplement 1B). We note that each of these analyses were performed with co-crystal structures of the somatic antibodies with $\mathrm{HA}$ (PDB ID: 4FOI (CR9114 -H5; CR9114 -H1 crystal structure not available) Dreyfus et al., 2012; 4FQY (CR9114 -H3) Dreyfus et al., 2012; 3GBN (CR6261 -H1) Ekiert et al., 2009).

\section{Pathway analysis}

Selection models

To study the likelihood of various mutational pathways leading from the germline to the somatic sequence, we must assume a selection model. Selection in germinal centers is considerably more complex than in classical population genetics models, involving spatial structure, changing population sizes, and T-cell-mediated selection, among other factors (Mesin et al., 2016). Capturing these aspects in quantitative models is an active field of research (Amitai et al., 2017). However, here we wish to adopt an extremely simple model of selection as a first step in understanding the impacts of the binding affinity landscape on antibody selection, with the goal of understanding the implications of the expectation that mutational steps become more probable as their effect on binding affinity becomes more positive. Combining the more realistic models of immune selection with our detailed characterization of mutational effects on antigen binding affinity remains an interesting avenue for future work.

Here, we restrict to the weak-mutation regime where mutation fixation events occur independently of one another. Selection proceeds as a Markov process, where the population is characterized by a single sequence that acquires a single mutation at each discrete step (McCandlish, 2011). We choose a simple form for the fixation probability of a mutation from sequence $s$ to sequence $t$, as discussed below. This then determines the transition probability for the population to move from $s$ to $t$. We assume that sequences cannot back-mutate (i.e. a residue changing from the somatic allele to the germline allele), and do not acquire multiple mutations in the same step. The absence of back-mutation is justified by the relatively large number of possible mutation sites compared to the total number of mutation events.

We define the transition probability of a single mutational step from the classical fixation probability for a mutation with selection coefficient $\sigma$ in a population of size $N$ (Kimura, 1962):

$$
p_{\text {step }}(\sigma, N)=\frac{1-e^{-\sigma}}{1-e^{-N \sigma}} \text {. }
$$


Here, we define the selection coefficient $\sigma$ to be proportional to the difference in log binding affinities to a particular antigen between the two sequences $s$ and $t$ :

$$
\sigma=\gamma \Delta_{s, t}^{\mathrm{ag}}=\gamma\left(-\log _{10} K_{D, t}^{\mathrm{ag}}-\left(-\log _{10} K_{D, s}^{\mathrm{ag}}\right)\right) .
$$

This model has two tunable parameters: $N$ represents the effective population size and $\gamma$ represents how strongly differences in binding affinity impact fitness. We chose three parameter values to span a range of selection strengths (see Figure 5-figure supplement 1): moderate, with $N=1000$ and $\gamma=1$; weak, with $N=20$ and $\gamma=0.5$; and strong, with $N \rightarrow \infty$ and $\gamma \rightarrow \infty$ such that $p_{\text {step }}$ reduces to a step function (one if $\Delta>0$ and 0 otherwise). These three models all show similar results, with differences between selection scenarios becoming more exaggerated with stronger selection and less exaggerated with weaker selection, as expected (see Figure 5-figure supplement 1).

From the fixation probabilities for a given parameter regime, we have the transition probability (up to a constant factor) for all sequences $s, t$ over all antigens ag,

$$
P_{s, t}^{\mathrm{ag}}= \begin{cases}p_{\mathrm{step}}\left(\Delta_{s, t}^{\mathrm{ag}}, \gamma, N\right) & \text { if } t \text { has one more somatic mutation than } s \\ 0 & \text { otherwise }\end{cases}
$$

which we use for all the calculations described below for results presented in Figure 5 and supplements.

\section{Scenario, mutation, and variant probabilities}

It is particularly useful to store the probabilities $P_{s, t}^{\mathrm{ag}}$ as (sparse) transition matrices $P^{\mathrm{ag}}$ of dimension $2^{N} \times 2^{N}$ for each antigen, where entries are nonzero only where sequence $t$ has one more somatic mutation than $s$.

First, we wish to obtain a measure of total probability for a particular antigen scenario, as shown in Figure 5E,F. We calculate this by computing the matrix product over all mutational steps $i$ for a particular sequence of antigen contexts $\left\{\operatorname{ag}_{1}, \ldots, \mathrm{ag}_{L}\right\}$ :

$$
\mathcal{P}_{\text {tot }}=\sum_{\text {paths }}\left(\prod_{\text {steps }} P_{\text {step }}\right)=\left[\prod_{i=1}^{L} P^{\mathrm{ag}_{i}}\right]_{s_{\mathrm{g}}, S_{\mathrm{s}}},
$$

where $[\cdot]_{s, s^{\prime}}$ corresponds to taking the matrix element in the row corresponding to variant $s$ and column corresponding to variant $s^{\prime}$. In the right-most term, the products are matrix operations and $s_{\mathrm{g}}, s_{\mathrm{S}}$ are respectively the indices of the germline and somatic variants.

We note that the transition probabilities $P^{\mathrm{ag}_{i}}$ are not normalized at each step. In practice, this means that mutations are optional: many outcomes will not reach the somatic sequence and the likelihood encodes the probability of reaching the somatic state. This makes it possible to compare different scenarios, as some scenarios are more likely than others to reach the somatic state. However, because these values do not represent true probabilities - the units are arbitrary - they cannot be compared between antibodies or between selection models. The exception is for the strong scenario, where the total probability for each path is one if all steps are uphill $\left(\Delta_{s, t}^{\mathrm{ag}}>0\right)$ and 0 otherwise. Thus, here $\mathcal{P}_{\text {tot }}$ has a natural interpretation as the total number of uphill paths. When we present results from the strong model (Figure 5C,D, Figure 5-figure supplement 1, and numbers of uphill paths for $\mathrm{H} 1$-only scenarios as discussed in the text), we represent uphill path numbers on a linear scale without log-transforming.

Although there are many possible antigen exposure scenarios, we restrict our analysis to several classes. First, in single-antigen scenarios, all steps $i$ use the same antigen. Second, for sequential scenarios, antigen exposures must occur in non-repeating segments (for example, $\mathrm{H} 1-\mathrm{H} 3-\mathrm{H} 1$ is not allowed), although we consider all possible lengths and orders of segments.

Mixed scenarios are more complicated, as we do not fully understand the nature of B cell interactions with multiple antigens in the same germinal center (Wang et al., 2015; Wang, 2017; Kuraoka et al., 2016). One option is to assume that the B cell engages the antigen for which it has the highest affinity and define $\Delta$ by the maximum binding affinity across all possible antigens at each step, but this definition would trivially imply that the mixed scenario has the highest probability. Instead, we choose two alternatives: first, 'average' mixed, where we assume the B cell engages all 
antigens and use the average binding affinity change over all three (for CR9114) or two (for CR6261) antigens, $\Delta_{\text {mixed }}=\frac{1}{N_{\mathrm{ag}}} \sum_{\mathrm{ag}} \Delta_{\mathrm{ag}}$; and second, 'random' mixed, where we assume the B cell randomly engages a single antigen and hence the antigen at each mutational step is chosen randomly. For the latter definition, we calculate $\mathcal{P}_{\text {tot }}$ as described above for 1000 randomly drawn scenarios and average the resulting log probability. When we illustrate mutational paths and mutation orders, we choose a representative scenario (with close to median probability) from the 1000 random draws.

We estimate the error of these probabilities by bootstrapping. Specifically, for 10 bootstrap iterations, we resample each binding affinity $-\log _{10} K_{D, s}^{\mathrm{ag}}$ from a normal distribution according to its value and standard deviation. We then recalculate the total probability $\mathcal{P}_{\text {tot }}$, average over the 10 values to obtain mean and s.e.m. values, and transform by the natural log for plotting, as shown in Figure 5 and Figure 5-figure supplement 1. We note that for the strong selection scenario (where probabilities represent total numbers of uphill paths), values are not log-transformed, and many scenarios have total path numbers of exactly zero. We refrain from studying the 'average' mixed scenario for strong selection because it is essentially equivalent to choosing the antigen with maximum improvement: the quantitative effect of averaging is undone when the transition probability is binarized. For CR6261, all mutations at the first mutational step are neutral (with the exception of one mutation that improves affinity for $\mathrm{H} 1$ only), and so we allow all mutations with equal probability for the first step in the strong selection model.

Next, to identify the most likely paths under a given exposure scenario, we reframe this Markov process as a directed weighted graph. Each sequence $s$ is a node, and a directed edge exists toward all sequences $t$ that can be reached by one additional somatic mutation. The edge weight is calculated from the transition probability, $w_{s \rightarrow t}=-\log \left(P_{s, t}^{\mathrm{ag}}+\epsilon\right)$, where $\epsilon$ is an extremely small value to ensure weights are finite. In this graph framework, we can use fast algorithms to obtain the 'shortest' paths from the germline to the somatic node (those for which the sum of weights is lowest, that is the total probability is highest). Specifically, we use the shortest_simple_paths function from the Python package networkx (Hagberg et al., 2008) to compute the $k$ shortest paths, as shown in Figure 5G,H. This method is exact and uses the algorithm described in Yen, 1971.

Next, we wish to obtain the probability that a mutation at site $m$ happened at a specific step $j$ (Figure $5 \mid, J)$. As we are focusing on one antigen context, we can normalize the transition matrices and define:

$$
\tilde{P}_{s, t}^{\mathrm{ag}}=P_{s, t}^{\mathrm{ag}} \times\left(\sum_{t} P_{s, t}^{\mathrm{ag}}\right)^{-1},
$$

if $P_{s, t}^{\mathrm{ag}} \neq 0$ and 0 otherwise. We can further restrict the transition matrix at step $j, \tilde{P}^{\mathrm{ag}_{j}}$, to have nonzero the mutation that occurs is at a particular residue $\alpha, \tilde{P}_{\alpha}^{\mathrm{ag}_{j}}$. The total relative probability for that site at that mutational step under an antigen exposure scenario is then

$$
\mathcal{P}_{j, \alpha}=\left[\left(\prod_{i=1}^{j-1} \tilde{P}^{\mathrm{ag}_{i}}\right) \cdot \tilde{P}_{\alpha}^{\mathrm{ag}_{j}} \cdot\left(\prod_{i=j+1}^{L} \tilde{P}^{\mathrm{ag}_{i}}\right)\right]_{s_{\mathrm{g}}, s_{\mathrm{s}}},
$$

where, again, products are matrix operations. Because a sequence of $L$ steps starting from the germline can only lead to the somatic state, $\tilde{P}$ verifies $\left[\prod_{i=1}^{L} \tilde{P}^{\mathrm{ag}_{i}}\right]_{s_{\mathrm{g}}, s_{\mathrm{s}}}=1$. With the relation $\sum_{\alpha} \tilde{P}_{\alpha}^{\mathrm{ag}_{j}}=\tilde{P}^{\mathrm{ag}_{j}}$ this implies that these probabilities are already normalized: $\sum_{\alpha} \mathcal{P}_{j, \alpha}=1$.

Finally, we wish to determine the total probability of each variant (Figure 5-figure supplement 2), that is the sum of probabilities of all paths passing through that variant, for a given selection scenario. For a variant $s$ that contains $j$ somatic mutations, we calculate

$$
\mathcal{P}_{s}=\left(\left[\prod_{i=1}^{j} \tilde{P}^{\mathrm{ag}_{i}}\right]_{s_{\mathrm{g}}, s}\right) \cdot\left(\left[\prod_{i=j+1}^{L} \tilde{P}^{\mathrm{ag}_{i}}\right]_{s, s_{\mathrm{s}}}\right),
$$

where the first term is the probability of reaching sequence $s$ at mutational step $j$, and the second term is the probability of reaching the somatic sequence after passing through sequence $s$. When representing this number we add an additional normalisation factor, $\mathcal{P}_{s}^{\prime}=\mathcal{P}_{s} \times n_{j}$, where $n_{j}=L j$ is the 
number of sequences with $j$ mutations, so that variants with different numbers of mutations have comparable values. $\mathcal{P}_{s}^{\prime}$ thus represents the ratio of the probability in a selective model to the probability in a neutral model (which is $1 / n_{j}$ ). Thus, sequences with $\log _{10}\left(\mathcal{P}_{s}^{\prime}\right)>0$ are favored by the given selection scenario, and those with $\log _{10}\left(\mathcal{P}_{s}^{\prime}\right)<0$ are disfavored, as shown in Figure 5-figure supplement 2 for moderate selection under the optimal sequential scenario.

\section{Acknowledgements}

We thank Rhys Adams for helpful discussion of the Tite-Seq experiments, Zach Niziolek for assistance with flow cytometry, Kevin McCarthy for help with antigen production, Matt Melissa for help acquiring strains and protocols, and Tyler Starr and members of the Denic, Gaudet, and Wittrup labs for help with experimental protocols. We also thank Jesse Bloom, Andrew Murray, and Michael Laub for helpful discussion and members of the Desai lab for comments on the manuscript. The computations in this paper were run on the FASRC Cannon cluster supported by the FAS Division of Science Research Computing Group at Harvard University.

\section{Additional information}

Competing interests

Aleksandra M Walczak: Senior editor, eLife. The other authors declare that no competing interests exist.

Funding

\begin{tabular}{|c|c|c|}
\hline Funder & Grant reference number & Author \\
\hline $\begin{array}{l}\text { Howard Hughes Medical Insti- } \\
\text { tute }\end{array}$ & $\begin{array}{l}\text { Hanna H. Gray Postdoctoral } \\
\text { Fellowship }\end{array}$ & Angela M Phillips \\
\hline Hertz Foundation & Graduate Fellowship Award & Katherine R Lawrence \\
\hline National Science Foundation & $\begin{array}{l}\text { Graduate Research } \\
\text { Fellowship Program }\end{array}$ & $\begin{array}{l}\text { Katherine R Lawrence } \\
\text { Jeffrey Chang } \\
\text { Milo S Johnson }\end{array}$ \\
\hline European Research Council & COG 724208 & $\begin{array}{l}\text { Thierry Mora } \\
\text { Aleksandra M Walczak }\end{array}$ \\
\hline National Institutes of Health & GM104239 & Michael M Desai \\
\hline National Science Foundation & PHY-1914916 & Michael M Desai \\
\hline Stanford University & Stanford Science Fellowship & Ivana Cvijovic \\
\hline $\begin{array}{l}\text { Human Frontier Science Pro- } \\
\text { gram }\end{array}$ & & Thomas Dupic \\
\hline $\begin{array}{l}\text { NSF-Simons Center for Math- } \\
\text { ematical and Statistical Analy- } \\
\text { sis of Biology at Harvard }\end{array}$ & 1764269 & Katherine R Lawrence \\
\hline
\end{tabular}

The funders had no role in study design, data collection and interpretation, or the decision to submit the work for publication.

Author contributions

Angela M Phillips, Katherine R Lawrence, Conceptualization, Software, Formal analysis, Supervision, Validation, Investigation, Methodology, Writing - original draft, Writing - review and editing; Alief Moulana, Software, Formal analysis, Validation, Investigation, Writing - review and editing; Thomas Dupic, Software, Formal analysis, Methodology, Writing - review and editing; Jeffrey Chang, Formal analysis, Validation, Investigation, Writing - review and editing; Milo S Johnson, Software, Visualization, Writing - review and editing; Ivana Cvijovic, Conceptualization, Investigation, Methodology, Writing - review and editing; Thierry Mora, Aleksandra M Walczak, Michael M Desai, Conceptualization, Supervision, Funding acquisition, Writing - review and editing 
Author ORCIDs

Angela M Phillips (DiD https://orcid.org/0000-0002-9806-7574

Milo S Johnson (Di) https://orcid.org/0000-0003-0169-2494

Ivana Cvijovic (D) http://orcid.org/0000-0002-6272-2979

Thierry Mora (iD) http://orcid.org/0000-0002-5456-9361

Aleksandra M Walczak (D) http://orcid.org/0000-0002-2686-5702

Michael M Desai (iD https://orcid.org/0000-0002-9581-1150

Decision letter and Author response

Decision letter https://doi.org/10.7554/eLife.71393.sa1

Author response https://doi.org/10.7554/eLife.71393.sa2

\section{Additional files}

Supplementary files

- Supplementary file 1. Primer sequences for sequencing library preparation.

- Supplementary file 2. Fragment sequences for Golden Gate construction of the CR9114 library.

- Supplementary file 3. Primer sequences for Golden Gate construction of the CR6261 library.

- Supplementary file 4. Plasmid map of pCT302 with CR9114 germline sequence.

- Supplementary file 5. Plasmid map of pCT302 with CR9114 somatic sequence.

- Supplementary file 6. Plasmid map of pCT302 with CR6261 germline sequence.

- Supplementary file 7. Plasmid map of pCT302 with CR6261 somatic sequence.

- Supplementary file 8. Plasmid map of pFastBac with influenza A/New Caldeonia/1999 H1 ectodomain.

- Supplementary file 9. Plasmid map of pFastBac with influenza A/Hong Kong/1999 H9 ectodomain.

- Supplementary file 10. Plasmid map of pFastBac with influenza A/Wisconsin/2005 H3 ectodomain.

- Supplementary file 11. Plasmid map of pFastBac with influenza B/Ohio/2005 HA ectodomain.

- Supplementary file 12. Inferred CR9114 VH germline nucleotide sequence.

- Transparent reporting form

Data availability

Data and code used for this study are available at https://github.com/klawrence26/bnab-landscapes (copy archived at https://archive.softwareheritage.org/swh:1:rev:61c1673a101ea739d5b7e9b282f6bcfad41d7e90). CR9114 data are also available in an interactive data browser at https:// yodabrowser.netlify.app/yoda_browser/. FASTQ files from high-throughput sequencing have been deposited in the NCBI BioProject database with accession number PRJNA741613.

The following datasets were generated:

\begin{tabular}{|c|c|c|c|c|}
\hline Author(s) & Year & Dataset title & Dataset URL & $\begin{array}{l}\text { Database and } \\
\text { Identifier }\end{array}$ \\
\hline $\begin{array}{l}\text { Phillips AM, } \\
\text { Lawrence KR, } \\
\text { Moulana A, Dupic T, } \\
\text { Chang J, Johnson } \\
\text { MS, Cvijovic I, Mora } \\
\text { T, Walczak AM, } \\
\text { Desai MM }\end{array}$ & 2021 & $\begin{array}{l}\text { Binding affinity landscapes } \\
\text { constrain the evolution of broadly } \\
\text { neutralizing anti-influenza } \\
\text { antibodies }\end{array}$ & $\begin{array}{l}\text { https://www.ncbi.nlm. } \\
\text { nih.gov/bioproject/? } \\
\text { term=PRJNA741613 }\end{array}$ & $\begin{array}{l}\text { NCBI BioProject, } \\
\text { PRJNA741613 }\end{array}$ \\
\hline $\begin{array}{l}\text { Phillips AM, } \\
\text { Lawrence KR, } \\
\text { Moulana A, Dupic T, } \\
\text { Chang J, Johnson } \\
\text { MS, Cvijovic I, Mora } \\
\text { T, Walczak AM, }\end{array}$ & 2021 & CR9114 Tite-Seq KD Measurements & $\begin{array}{l}\text { https://yodabrowser.ne- } \\
\text { tlify.app/yoda_browser/ }\end{array}$ & $\begin{array}{l}\text { YODA online data } \\
\text { browser, CR9114 }\end{array}$ \\
\hline
\end{tabular}




\section{References}

Adams RM, Mora T, Walczak AM, Kinney JB. 2016. Measuring the sequence-affinity landscape of antibodies with massively parallel titration curves. eLife 5:e23156. DOI: https://doi.org/10.7554/eLife.23156, PMID: 28035901

Adams RM, Kinney JB, Walczak AM, Mora T. 2019. Epistasis in a fitness landscape defined by Antibody-Antigen binding free energy. Cell Systems 8:86-93. DOI: https://doi.org/10.1016/j.cels.2018.12.004, PMID: 30611676

Amitai A, Mesin L, Victora GD, Kardar M, Chakraborty AK. 2017. A population dynamics model for clonal diversity in a germinal center. Frontiers in Microbiology 8:1693. DOI: https://doi.org/10.3389/fmicb.2017. 01693, PMID: 28955307

Avnir Y, Tallarico AS, Zhu Q, Bennett AS, Connelly G, Sheehan J, Sui J, Fahmy A, Huang CY, Cadwell G, Bankston LA, McGuire AT, Stamatatos L, Wagner G, Liddington RC, Marasco WA. 2014. Molecular signatures of hemagglutinin stem-directed heterosubtypic human neutralizing antibodies against influenza $A$ viruses. PLOS Pathogens 10:e1004103. DOI: https://doi.org/10.1371/journal.ppat.1004103, PMID: 24788925

Bajic G, van der Poel CE, Kuraoka M, Schmidt AG, Carroll MC, Kelsoe G, Harrison SC. 2019. Autoreactivity profiles of influenza hemagglutinin broadly neutralizing antibodies. Scientific Reports 9:3492. DOI: https://doi. org/10.1038/s41598-019-40175-8, PMID: 30837606

Baker EN, Hubbard RE. 1984. Hydrogen bonding in globular proteins. Progress in Biophysics and Molecular Biology 44:97-179. DOI: https://doi.org/10.1016/0079-6107(84)90007-5, PMID: 6385134

Bank C, Hietpas RT, Jensen JD, Bolon DN. 2015. A systematic survey of an intragenic epistatic landscape. Molecular Biology and Evolution 32:229-238. DOI: https://doi.org/10.1093/molbev/msu301, PMID: 25371431

Barnett M. 2013. Regex. https://pypi.org/project/regex/

Batista FD, Neuberger MS. 1998. Affinity dependence of the B cell response to antigen: a threshold, a ceiling, and the importance of off-rate. Immunity 8:751-759. DOI: https://doi.org/10.1016/S1074-7613(00)80580-4, PMID: 9655489

Boder ET, Wittrup KD. 1997. Yeast surface display for screening combinatorial polypeptide libraries. Nature Biotechnology 15:553-557. DOI: https://doi.org/10.1038/nbt0697-553, PMID: 9181578

Bondi A. 1964. van der Waals Volumes and Radii. The Journal of Physical Chemistry 68:441-451. DOI: https:// doi.org/10.1021/j100785a001

Braden BC, Goldman ER, Mariuzza RA, Poljak RJ. 1998. Anatomy of an antibody molecule: structure, kinetics, thermodynamics and mutational studies of the antilysozyme antibody D1.3. Immunological Reviews 163:45-57. DOI: https://doi.org/10.1111/j.1600-065X.1998.tb01187.x, PMID: 9700501

Burks EA, Chen G, Georgiou G, Iverson BL. 1997. In vitro scanning saturation mutagenesis of an antibody binding pocket. PNAS 94:412-417. DOI: https://doi.org/10.1073/pnas.94.2.412, PMID: 9012796

Chen G, Dubrawsky I, Mendez P, Georgiou G, Iverson BL. 1999. In vitro scanning saturation mutagenesis of all the specificity determining residues in an antibody binding site. Protein Engineering, Design and Selection 12: 349-356. DOl: https://doi.org/10.1093/protein/12.4.349, PMID: 10325406

Corti D, Voss J, Gamblin SJ, Codoni G, Macagno A, Jarrossay D, Vachieri SG, Pinna D, Minola A, Vanzetta F, Silacci C, Fernandez-Rodriguez BM, Agatic G, Bianchi S, Giacchetto-Sasselli I, Calder L, Sallusto F, Collins P, Haire LF, Temperton N, et al. 2011. A neutralizing antibody selected from plasma cells that binds to group 1 and group 2 influenza A hemagglutinins. Science 333:850-856. DOI: https://doi.org/10.1126/science.1205669, PMID: 21798894

Corti D, Cameroni E, Guarino B, Kallewaard NL, Zhu Q, Lanzavecchia A. 2017. Tackling influenza with broadly neutralizing antibodies. Current Opinion in Virology 24:60-69. DOI: https://doi.org/10.1016/j.coviro.2017.03. 002, PMID: 28527859

Corti D, Lanzavecchia A. 2013. Broadly neutralizing antiviral antibodies. Annual Review of Immunology 31:705742. DOI: https://doi.org/10.1146/annurev-immunol-032712-095916, PMID: 23330954

Domingo J, Baeza-Centurion P, Lehner B. 2019. The causes and consequences of genetic interactions (Epistasis). Annual Review of Genomics and Human Genetics 20:433-460. DOI: https://doi.org/10.1146/annurev-genom083118-014857, PMID: 31082279

Doud MB, Lee JM, Bloom JD. 2018. How single mutations affect viral escape from broad and narrow antibodies to $\mathrm{H} 1$ influenza hemagglutinin. Nature Communications 9:1386. DOI: https://doi.org/10.1038/s41467-01803665-3, PMID: 29643370

Dreyfus C, Laursen NS, Kwaks T, Zuijdgeest D, Khayat R, Ekiert DC, Lee JH, Metlagel Z, Bujny MV, Jongeneelen $M$, van der Vlugt R, Lamrani M, Korse HJ, Geelen E, Sahin Ö, Sieuwerts M, Brakenhoff JP, Vogels R, Li OT, Poon LL, et al. 2012. Highly conserved protective epitopes on influenza B viruses. Science 337:1343-1348. DOI: https://doi.org/10.1126/science.1222908, PMID: 22878502

Ekiert DC, Bhabha G, Elsliger MA, Friesen RH, Jongeneelen M, Throsby M, Goudsmit J, Wilson IA. 2009. Antibody recognition of a highly conserved influenza virus epitope. Science 324:246-251. DOI: https://doi.org/ 10.1126/science.1171491, PMID: 19251591

Ekiert DC, Friesen RH, Bhabha G, Kwaks T, Jongeneelen M, Yu W, Ophorst C, Cox F, Korse HJ, Brandenburg B, Vogels R, Brakenhoff JP, Kompier R, Koldijk MH, Cornelissen LA, Poon LL, Peiris M, Koudstaal W, Wilson IA, Goudsmit J. 2011. A highly conserved neutralizing epitope on group 2 influenza A viruses. Science 333:843850. DOI: https://doi.org/10.1126/science.1204839, PMID: 21737702 
Engler C, Kandzia R, Marillonnet S. 2008. A one pot, one step, precision cloning method with high throughput capability. PLOS ONE 3:e3647. DOI: https://doi.org/10.1371/journal.pone.0003647, PMID: 18985154

Esmaielbeiki R, Krawczyk K, Knapp B, Nebel JC, Deane CM. 2016. Progress and challenges in predicting protein interfaces. Briefings in Bioinformatics 17:117-131. DOl: https://doi.org/10.1093/bib/bbv027, PMID: 25971595

Fairhead M, Howarth M. 2015. Site-specific biotinylation of purified proteins using BirA. In: Howarth M (Ed). SiteSpecific Protein Labeling. Germany: Springer. p. 171-184. DOI: https://doi.org/10.1007/978-1-4939-2272-7

Forsyth CM, Juan V, Akamatsu Y, DuBridge RB, Doan M, Ivanov AV, Ma Z, Polakoff D, Razo J, Wilson K, Powers DB. 2013. Deep mutational scanning of an antibody against epidermal growth factor receptor using mammalian cell display and massively parallel pyrosequencing. mAbs 5:523-532. DOI: https://doi.org/10.4161/ mabs.24979, PMID: 23765106

Gibson DG, Young L, Chuang RY, Venter JC, Hutchison CA, Smith HO. 2009. Enzymatic assembly of DNA molecules up to several hundred kilobases. Nature Methods 6:343-345. DOI: https://doi.org/10.1038/nmeth. 1318, PMID: 19363495

Gietz RD, Schiestl RH. 2007. High-efficiency yeast transformation using the LiAc/SS carrier DNA/PEG method. Nature Protocols 2:31-34. DOI: https://doi.org/10.1038/nprot.2007.13, PMID: 17401334

Giudicelli V, Duroux P, Ginestoux C, Folch G, Jabado-Michaloud J, Chaume D, Lefranc MP. 2006. IMGT/LIGMDB, the IMGT comprehensive database of immunoglobulin and T cell receptor nucleotide sequences. Nucleic Acids Research 34:D781-D784. DOI: https://doi.org/10.1093/nar/gkj088, PMID: 16381979

Gong LI, Suchard MA, Bloom JD. 2013. Stability-mediated epistasis constrains the evolution of an influenza protein. eLife 2:e00631. DOI: https://doi.org/10.7554/eLife.00631, PMID: 23682315

Guthmiller JJ, Lan LY, Fernández-Quintero ML, Han J, Utset HA, Bitar DJ, Hamel NJ, Stovicek O, Li L, Tepora M, Henry C, Neu KE, Dugan HL, Borowska MT, Chen YQ, Liu STH, Stamper CT, Zheng NY, Huang M, Palm AE, et al. 2020. Polyreactive broadly neutralizing B cells are selected to provide defense against pandemic threat influenza viruses. Immunity 53:1230-1244. DOI: https://doi.org/10.1016/j.immuni.2020.10.005, PMID: 330 96040

Hagberg AA, Schult DA, Swart PJ. 2008. Exploring network structure, dynamics, and function using NetworkX. Proceedings of the 7th Python in Science Conference 11-15. https://www.osti.gov/biblio/960616-exploringnetwork-structure-dynamics-function-using-networkx.

Henry C, Palm AE, Krammer F, Wilson PC. 2018. From original antigenic sin to the universal influenza virus vaccine. Trends in Immunology 39:70-79. DOI: https://doi.org/10.1016/j.it.2017.08.003, PMID: 28867526

Howarth M, Takao K, Hayashi Y, Ting AY. 2005. Targeting quantum dots to surface proteins in living cells with biotin ligase. PNAS 102:7583-7588. DOI: https://doi.org/10.1073/pnas.0503125102, PMID: 15897449

Israelachvili J, Pashley R. 1982. The hydrophobic interaction is long range, decaying exponentially with distance. Nature 300:341-342. DOI: https://doi.org/10.1038/300341a0, PMID: 7144887

Kimura M. 1962. On the probability of fixation of mutant genes in a population. Genetics 47:713-719. DOI: https://doi.org/10.1093/genetics/47.6.713, PMID: 14456043

Klein F, Diskin R, Scheid JF, Gaebler C, Mouquet H, Georgiev IS, Pancera M, Zhou T, Incesu RB, Fu BZ, Gnanapragasam PN, Oliveira TY, Seaman MS, Kwong PD, Bjorkman PJ, Nussenzweig MC. 2013. Somatic mutations of the immunoglobulin framework are generally required for broad and potent HIV-1 neutralization. Cell 153:126-138. DOI: https://doi.org/10.1016/j.cell.2013.03.018, PMID: 23540694

Koenig P, Lee CV, Walters BT, Janakiraman V, Stinson J, Patapoff TW, Fuh G. 2017. Mutational landscape of antibody variable domains reveals a switch modulating the interdomain conformational dynamics and antigen binding. PNAS 114:E486-E495. DOI: https://doi.org/10.1073/pnas.1613231114, PMID: 28057863

Krammer F, Pica N, Hai R, Tan GS, Palese P. 2012. Hemagglutinin Stalk-Reactive antibodies are boosted following sequential infection with seasonal and pandemic H1N1 influenza virus in mice. Journal of Virology 86: 10302-10307. DOI: https://doi.org/10.1128/JVI.01336-12, PMID: 22787225

Kuraoka M, Schmidt AG, Nojima T, Feng F, Watanabe A, Kitamura D, Harrison SC, Kepler TB, Kelsoe G. 2016. Complex antigens drive permissive clonal selection in germinal centers. Immunity 44:542-552. DOI: https://doi. org/10.1016/j.immuni.2016.02.010, PMID: 26948373

Lingwood D, McTamney PM, Yassine HM, Whittle JR, Guo X, Boyington JC, Wei CJ, Nabel GJ. 2012. Structural and genetic basis for development of broadly neutralizing influenza antibodies. Nature 489:566-570. DOI: https://doi.org/10.1038/nature11371, PMID: 22932267

Madan B, Zhang B, Xu K, Chao CW, O'Dell S, Wolfe JR, Chuang GY, Fahad AS, Geng H, Kong R, Louder MK, Nguyen TD, Rawi R, Schön A, Sheng Z, Nimrania R, Wang Y, Zhou T, Lin BC, Doria-Rose NA, et al. 2021. Mutational fitness landscapes reveal genetic and structural improvement pathways for a vaccine-elicited HIV-1 broadly neutralizing antibody. PNAS 118:e2011653118. DOI: https://doi.org/10.1073/pnas.2011653118, PMID: 33649208

Margine I, Palese P, Krammer F. 2013. Expression of functional recombinant hemagglutinin and neuraminidase proteins from the novel H7N9 influenza virus using the baculovirus expression system. Journal of Visualized Experiments 81:e51112. DOI: https://doi.org/10.3791/51112

McCandlish DM. 2011. Visualizing fitness landscapes. Evolution 65:1544-1558. DOI: https://doi.org/10.1111/j. 1558-5646.2011.01236.x, PMID: 21644947

Mesin L, Ersching J, Victora GD. 2016. Germinal Center B Cell Dynamics. Immunity 45:471-482. DOI: https://doi. org/10.1016/j.immuni.2016.09.001, PMID: 27653600

Midelfort KS, Hernandez HH, Lippow SM, Tidor B, Drennan CL, Wittrup KD. 2004. Substantial energetic improvement with minimal structural perturbation in a high affinity mutant antibody. Journal of Molecular Biology 343:685-701. DOI: https://doi.org/10.1016/j.jmb.2004.08.019, PMID: 15465055 
Miton CM, Tokuriki N. 2016. How mutational epistasis impairs predictability in protein evolution and design.

Protein Science 25:1260-1272. DOI: https://doi.org/10.1002/pro.2876, PMID: 26757214

Molari M, Eyer K, Baudry J, Cocco S, Monasson R. 2020. Quantitative modeling of the effect of antigen dosage on B-cell affinity distributions in maturating germinal centers. eLife 9:e55678. DOI: https://doi.org/10.7554/ eLife.55678, PMID: 32538783

Nguyen Ba AN, Cvijović I, Rojas Echenique Jl, Lawrence KR, Rego-Costa A, Liu X, Levy SF, Desai MM. 2019. High-resolution lineage tracking reveals travelling wave of adaptation in laboratory yeast. Nature 575:494-499. DOI: https://doi.org/10.1038/s41586-019-1749-3, PMID: 31723263

Olson CA, Wu NC, Sun R. 2014. A comprehensive biophysical description of pairwise epistasis throughout an entire protein domain. Current Biology 24:2643-2651. DOI: https://doi.org/10.1016/j.cub.2014.09.072, PMID: 25455030

Ortlund EA, Bridgham JT, Redinbo MR, Thornton JW. 2007. Crystal structure of an ancient protein: evolution by conformational epistasis. Science 317:1544-1548. DOI: https://doi.org/10.1126/science.1142819, PMID: 17702 911

Otwinowski J. 2018. Biophysical Inference of Epistasis and the Effects of Mutations on Protein Stability and Function. Molecular Biology and Evolution 35:2345-2354. DOI: https://oi.org/10.1093/molbev/msy141, PMID: 30085303

Otwinowski J, McCandlish DM, Plotkin JB. 2018. Inferring the shape of global epistasis. PNAS 115:E7550E7558. DOI: https://doi.org/10.1073/pnas.1804015115, PMID: 30037990

Pappas L, Foglierini M, Piccoli L, Kallewaard NL, Turrini F, Silacci C, Fernandez-Rodriguez B, Agatic G, Giacchetto-Sasselli I, Pellicciotta G, Sallusto F, Zhu Q, Vicenzi E, Corti D, Lanzavecchia A. 2014. Rapid development of broadly influenza neutralizing antibodies through redundant mutations. Nature 516:418-422. DOI: https://doi.org/10.1038/nature13764, PMID: 25296253

Peterman N, Levine E. 2016. Sort-seq under the hood: implications of design choices on large-scale characterization of sequence-function relations. BMC Genomics 17:206. DOI: https://doi.org/10.1186/s12864016-2533-5, PMID: 26956374

Pettersen EF, Goddard TD, Huang CC, Meng EC, Couch GS, Croll TI, Morris JH, Ferrin TE. 2021. UCSF ChimeraX: Structure visualization for researchers, educators, and developers. Protein Science 30:70-82. DOI: https://doi.org/10.1002/pro.3943, PMID: 32881101

Phillips A. 2021. bnab-landscapes. Software Heritage. swh:1:rev:61c1673a101ea739d5b7e9b282f6bcfad41d7e90 https://archive.softwareheritage.org/swh:1:rev:61c1673a101ea739d5b7e9b282f6bcfad41d7e90.

Podgornaia Al, Laub MT. 2015. Protein evolution Pervasive degeneracy and epistasis in a protein-protein interface. Science 347:673-677. DOI: https://doi.org/10.1126/science.1257360, PMID: 25657251

Poelwijk FJ, Krishna V, Ranganathan R. 2016. The Context-Dependence of mutations: a linkage of formalisms. PLOS Computational Biology 12:e1004771. DOI: https://doi.org/10.1371/journal.pcbi.1004771, PMID: 273376 95

Poelwijk FJ, Socolich M, Ranganathan R. 2019. Learning the pattern of epistasis linking genotype and phenotype in a protein. Nature Communications 10:4213. DOI: https://doi.org/10.1038/s41467-019-12130-8, PMID: 31527666

Rotem A, Serohijos AWR, Chang CB, Wolfe JT, Fischer AE, Mehoke TS, Zhang H, Tao Y, Lloyd Ung W, Choi JM, Rodrigues JV, Kolawole AO, Koehler SA, Wu S, Thielen PM, Cui N, Demirev PA, Giacobbi NS, Julian TR, Schwab K, et al. 2018. Evolution on the biophysical fitness landscape of an RNA virus. Molecular Biology and Evolution 35:2390-2400. DOI: https://doi.org/10.1093/molbev/msy131, PMID: 29955873

Sachdeva V, Husain K, Sheng J, Wang S, Murugan A. 2020. Tuning environmental timescales to evolve and maintain generalists. PNAS 117:12693-12699. DOI: https://doi.org/10.1073/pnas.1914586117, PMID: 32457160

Sailer ZR, Harms MJ. 2017a. Detecting High-Order epistasis in nonlinear Genotype-Phenotype maps. Genetics 205:1079-1088. DOI: https://doi.org/10.1534/genetics.116.195214, PMID: 28100592

Sailer ZR, Harms MJ. 2017b. High-order epistasis shapes evolutionary trajectories. PLOS Computational Biology 13:e1005541. DOI: https://doi.org/10.1371/journal.pcbi.1005541, PMID: 28505183

Sarkisyan KS, Bolotin DA, Meer MV, Usmanova DR, Mishin AS, Sharonov GV, Ivankov DN, Bozhanova NG, Baranov MS, Soylemez O, Bogatyreva NS, Vlasov PK, Egorov ES, Logacheva MD, Kondrashov AS, Chudakov DM, Putintseva EV, Mamedov IZ, Tawfik DS, Lukyanov KA, et al. 2016. Local fitness landscape of the green fluorescent protein. Nature 533:397-401. DOI: https://doi.org/10.1038/nature17995, PMID: 27193686

Schmidt AG, Do KT, McCarthy KR, Kepler TB, Liao HX, Moody MA, Haynes BF, Harrison SC. 2015. Immunogenic stimulus for germline precursors of antibodies that engage the influenza hemagglutinin Receptor-Binding site. Cell Reports 13:2842-2850. DOI: https://doi.org/10.1016/j.celrep.2015.11.063, PMID: 26711348

Schrodinger, LLC. 2015. The PyMOL Molecular Graphics System. 1.8.

Smith DJ, Lapedes AS, de Jong JC, Bestebroer TM, Rimmelzwaan GF, Osterhaus AD, Fouchier RA. 2004. Mapping the antigenic and genetic evolution of influenza virus. Science 305:371-376. DOI: https://doi.org/10. 1126/science.1097211, PMID: 15218094

Spisak N, Walczak AM, Mora T. 2020. Learning the heterogeneous hypermutation landscape of immunoglobulins from high-throughput repertoire data. Nucleic Acids Research 48:10702-10712. DOI: https://doi.org/10.1093/ nar/gkaa825, PMID: 33035336

Sprenger KG, Louveau JE, Murugan PM, Chakraborty AK. 2020. Optimizing immunization protocols to elicit broadly neutralizing antibodies. PNAS 117:20077-20087. DOI: https://doi.org/10.1073/pnas.1919329117, PMID: 32747563 
Starr TN, Picton LK, Thornton JW. 2017. Alternative evolutionary histories in the sequence space of an ancient protein. Nature 549:409-413. DOl: https://doi.org/10.1038/nature23902, PMID: 28902834

Starr TN, Greaney AJ, Addetia A, Hannon WW, Choudhary MC, Dingens AS, Li JZ, Bloom JD. 2021. Prospective mapping of viral mutations that escape antibodies used to treat COVID-19. Science 371:850-854. DOI: https:// doi.org/10.1126/science.abf9302, PMID: 33495308

Throsby M, van den Brink E, Jongeneelen M, Poon LL, Alard P, Cornelissen L, Bakker A, Cox F, van Deventer E, Guan Y, Cinatl J, ter Meulen J, Lasters I, Carsetti R, Peiris M, de Kruif J, Goudsmit J. 2008. Heterosubtypic neutralizing monoclonal antibodies cross-protective against $\mathrm{H} 5 \mathrm{~N} 1$ and $\mathrm{H} 1 \mathrm{~N} 1$ recovered from human IgM+ memory B cells. PLOS ONE 3:e3942. DOl: https://doi.org/10.1371/journal.pone.0003942, PMID: 19079604

Unniraman S, Schatz DG. 2007. Strand-biased spreading of mutations during somatic hypermutation. Science 317:1227-1230. DOI: https://doi.org/10.1126/science.1145065, PMID: 17761884

Victora GD, Nussenzweig MC. 2012. Germinal centers. Annual Review of Immunology 30:429-457. DOI: https:// doi.org/10.1146/annurev-immunol-020711-075032, PMID: 22224772

Wang TT, Tan GS, Hai R, Pica N, Petersen E, Moran TM, Palese P. 2010. Broadly protective monoclonal antibodies against $\mathrm{H} 3$ influenza viruses following sequential immunization with different hemagglutinins. PLOS Pathogens 6:e1000796. DOI: https://doi.org/10.1371/journal.ppat.1000796, PMID: 20195520

Wang S, Mata-Fink J, Kriegsman B, Hanson M, Irvine DJ, Eisen HN, Burton DR, Wittrup KD, Kardar M, Chakraborty AK. 2015. Manipulating the selection forces during affinity maturation to generate cross-reactive HIV antibodies. Cell 160:785-797. DOI: https://doi.org/10.1016/j.cell.2015.01.027, PMID: 25662010

Wang S. 2017. Optimal sequential immunization can focus antibody responses against diversity loss and distraction. PLOS Computational Biology 13:e1005336. DOI: https://doi.org/10.1371/journal.pcbi.1005336, PMID: 28135270

Weinreich DM, Delaney NF, Depristo MA, Hartl DL. 2006. Darwinian evolution can follow only very few mutational paths to fitter proteins. Science 312:111-114. DOI: https://doi.org/10.1126/science.1123539, PMID: 16601193

Wells JA. 1990. Additivity of mutational effects in proteins. Biochemistry 29:8509-8517. DOI: https://doi.org/10. 1021/bi00489a001, PMID: 2271534

Wiley DC, Wilson IA, Skehel JJ. 1981. Structural identification of the antibody-binding sites of Hong Kong influenza haemagglutinin and their involvement in antigenic variation. Nature 289:373-378. DOI: https://doi. org/10.1038/289373a0, PMID: 6162101

Wu NC, Grande G, Turner HL, Ward AB, Xie J, Lerner RA, Wilson IA. 2017. In vitro evolution of an influenza broadly neutralizing antibody is modulated by hemagglutinin receptor specificity. Nature Communications 8 15371. DOI: https://doi.org/10.1038/ncomms15371, PMID: 28504265

Wu NC, Thompson AJ, Lee JM, Su W, Arlian BM, Xie J, Lerner RA, Yen HL, Bloom JD, Wilson IA. 2020. Different genetic barriers for resistance to HA stem antibodies in influenza $\mathrm{H} 3$ and $\mathrm{H} 1$ viruses. Science 368:1335-1340. DOI: https://doi.org/10.1126/science.aaz5143, PMID: 32554590

Xu H, Schmidt AG, O'Donnell T, Therkelsen MD, Kepler TB, Moody MA, Haynes BF, Liao HX, Harrison SC, Shaw DE. 2015. Key mutations stabilize antigen-binding conformation during affinity maturation of a broadly neutralizing influenza antibody lineage. Proteins: Structure, Function, and Bioinformatics 83:771-780. DOI: https://doi.org/10.1002/prot.24745, PMID: 25524709

Ye J, Ma N, Madden TL, Ostell JM. 2013. IgBLAST: an immunoglobulin variable domain sequence analysis tool. Nucleic Acids Research 41:W34-W40. DOI: https://doi.org/10.1093/nar/gkt382, PMID: 23671333

Yen JY. 1971. Finding the K shortest loopless paths in a network. Management Science 17:712-716. DOI: https://doi.org/10.1287/mnsc.17.11.712

Yewdell JW. 2013. To dream the impossible dream: universal influenza vaccination. Current Opinion in Virology 3:316-321. DOI: https://doi.org/10.1016/j.coviro.2013.05.008, PMID: 23835048 


\section{Appendix 1}

\section{Maximum likelihood approach to binding affinity inference}

In this approach we make the assumption that the fluorescence emitted by cells of a specific genotype is distributed log-normally, with parameters $\mu_{s, c}$ and $\sigma_{s, c}$ (the mean and standard deviation of the associated normal distribution respectively). At concentration $c$, a cell with genotype $s$ will fall into the bin $b$ ( $\log _{10}$-fluorescence values $f_{s, c}$ ranging from $l_{b}$ to $\left.h_{b}\right)$ with probability:

$$
\begin{gathered}
P\left[l_{b}<f_{s, c}<h_{b}\right]=\int_{l_{b}}^{h_{b}} \frac{1}{\sqrt{2 \pi \sigma_{s, c}^{2}}} e^{-\frac{1}{2}\left(\frac{f_{s, c}-\mu_{s, c}}{\sigma_{s, c}}\right)^{2}} \mathrm{~d} f_{s, c} \\
=\frac{1}{2}\left(\operatorname{erf}\left(\frac{h_{b}-\mu_{s, c}}{\sigma_{s, c} \sqrt{2}}\right)-\operatorname{erf}\left(\frac{l_{b}-\mu_{s, c}}{\sigma_{s, c} \sqrt{2}}\right)\right) .
\end{gathered}
$$

Each cell sorted is an independent event, so the number of cells in each bin will be multinomially distributed, and thus the likelihood of sorting $n_{b, s \mid c}$ cells of sequence $s$ into bin $b$ at concentration $c$ is given by

$$
\mathcal{L}=\prod_{s, c}\left(P\left[l_{b}<f_{s, c}<h_{b}\right]\right)^{n_{b, s \mid c}}
$$

and the log-likelihood is

$$
\log \mathcal{L}=\sum_{s, c, b} n_{b, s \mid c} \log P\left[l_{b}<f_{s, c}<h_{b}\right] \propto \sum_{s, c, b} p_{b, s \mid c} \log P\left[l_{b}<f_{s, c}<h_{b}\right] .
$$

The probability $p_{b, s \mid c}$ is estimated as in the mean-bin approach (see Methods) and the log-likelihood is then maximized as a function of $\mu_{s, c}$ and $\sigma_{s, c}$ (BFGS method). The values of $A, K_{D}$, and $B$ are then estimated similarly as the mean-bin approach (see Methods), replacing $\bar{F}_{s, c}$ by $\mu_{s, c}$.

The $-\log _{10} K_{D}$ inferred by this maximum likelihood $(\mathrm{ML})$ approach correlate well with isogenic flow cytometry $-\log _{10} K_{D}$ (see Appendix 1-figure 1), but not as well as those inferred by the mean-bin approach (Figure 1-figure supplement 2B). The ML approach is predicated on the assumption that the fluorescence distribution for each variant is log-normal, which is often not the case (see Appendix 1-figure 2). For these reasons, in addition to favoring a simple approach, we performed all analyses with $-\log _{10} K_{D}$ inferred by the mean-bin approach.

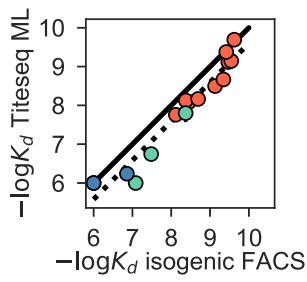

Appendix 1-figure 1. Correlation between - $\log _{10} K_{D}$ from $\mathrm{ML}$ inference on Tite-Seq data vs. $-\log _{10} K_{D}$ from isogenic flow cytometry. $-\log _{10} K_{D}$ to $\mathrm{H} 1$ (salmon), $\mathrm{H} 3$ (green), and Flu B (blue) shown for select variants, identical to those shown in Figure 1-figure supplement 2B. Pearson's $r$ $=0.97$. 

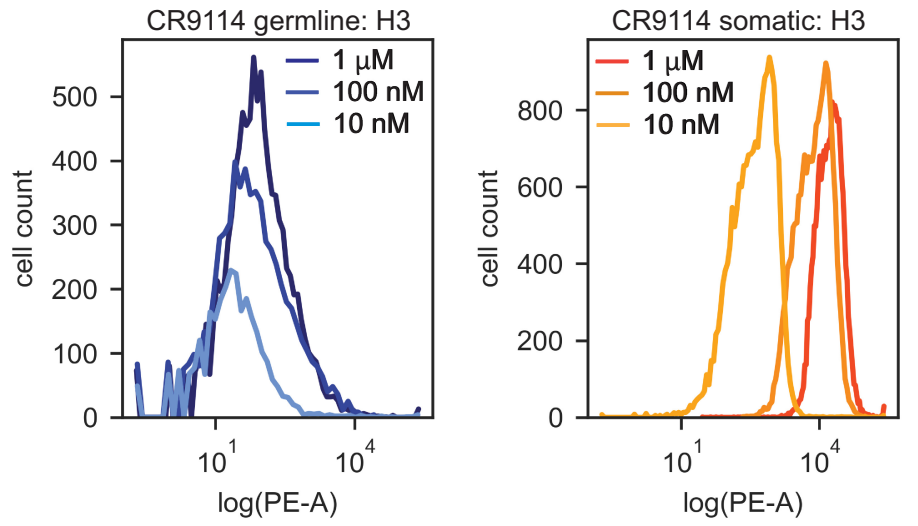

Appendix 1-figure 2. Distributions of PE-A fluorescence (HA binding) for isogenic CR9114 strains incubated with H3. PE-A fluorescence distributions from flow cytometry of isogenic CR9114 germline (left) and somatic (right) strains following incubation with $1 \mu \mathrm{M}, 100 \mathrm{nM}$, and $10 \mathrm{nM} \mathrm{H3}$, as described in Methods. Shape of distribution varies for different clones and is not strictly log-normal, hence deviating from assumptions made in the maximum-likelihood binding affinity inference. 


\section{Appendix 2}

\section{Alternative approaches to epistasis inference}

\section{Statistical epistasis and variance partitioning}

The contrast between biochemical and statistical frameworks for epistasis is well described in Poelwijk et al., 2016. In particular, a biochemical epistasis approach highlights one particular sequence as the "wildtype" or reference sequence and measures effects relative to its phenotype, whereas a statistical epistasis approach measures effects relative to the average phenotype of all variants included. The biochemical approach benefits from easier interpretation of the coefficient values, particularly when there is a natural or relevant choice of reference sequence, but the coefficients at different orders are not statistically independent. The statistical approach allows for correct variance partitioning between interaction orders, but the interpretation of the coefficients can be sensitive to the set of sequences, particularly when not all possible sequences are represented or when a majority of sequences exhibit some uninteresting phenotype (e.g. lethal).

Here, we perform inference of statistical epistasis exactly as described above for biochemical epistasis (see Methods), except that genotypes $x_{i, s}$ are coded as $\{-1,1\}$ instead of $\{0,1\}$. The results from this statistical epistasis inference are shown in Appendix 2-figure 1 for CR9114 and Appendix 2-figure 2 for CR6261, in plots analogous to those in Figure 3, Figure 4 and supplements. We find that the patterns of site participation in interactions are similar (although the coefficient magnitudes and signs are of course scaled differently). The group of five key sites discussed in Figure 3 (sites 30, 57, 65, 82, and 83 for CR9114 binding to H1) exhibit coefficients that are significant for all 31 mutation combinations, consistent with the result from biochemical epistasis. Overall, the numbers of significant coefficients inferred in statistical epistasis models tends to be somewhat higher than for biochemical epistasis models, perhaps due to the effect of background averaging in reducing coefficient standard errors, but neither framework is a substantially more compact representation of epistasis than the other.

In the statistical epistasis framework, we can also partition the variance explained by the model according to the interaction order. Here, we take the final inferred model at the optimal interaction order and evaluate the prediction performance $\left(R^{2}\right)$ of each order as a fraction of the total performance of the full model. As shown in Appendix 2-figure 3, we find that epistasis explains a substantial fraction of variance (18\%-33\%, depending on antibody-antigen pair). Variance explained tends to decline with increasing order, as is also observed in some other protein epistasis datasets (Sailer and Harms, 2017b). This indicates that interactions at higher order are more rare (compared to the total number of terms at each order, which scales combinatorially) and/or smaller in magnitude than those at lower order. However, this does not imply that rare, strong interactions of even higher order do not exist; for example, there may be some strong sixth-order interaction terms for CR9114 binding to $\mathrm{H} 1$, but not enough to compensate for the many nonsignificant sixth-order terms in our cross-validation framework. 
A

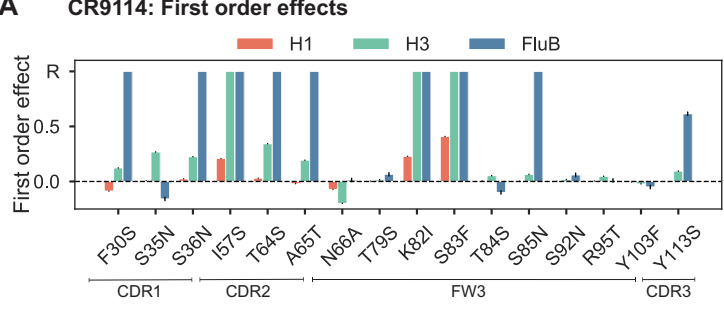

B CR9114: Second order effects

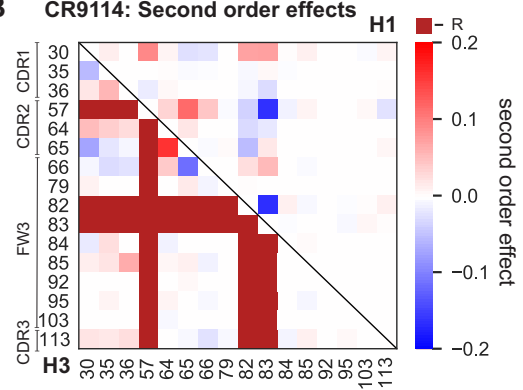

C CR9114 H1: $3^{\text {rd }}-5^{\text {th }}$ order effects

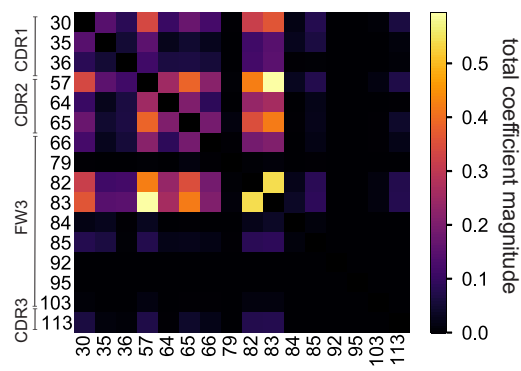

D CR9114 H3: $3^{\text {rd }}-4^{\text {th }}$ order effects

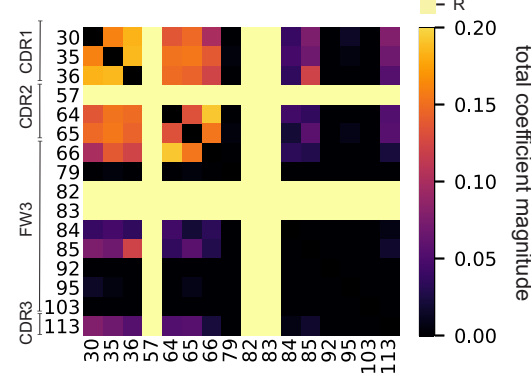

E CR9114 H1: coefficients for interactions between $30,57,65,82$, and 83
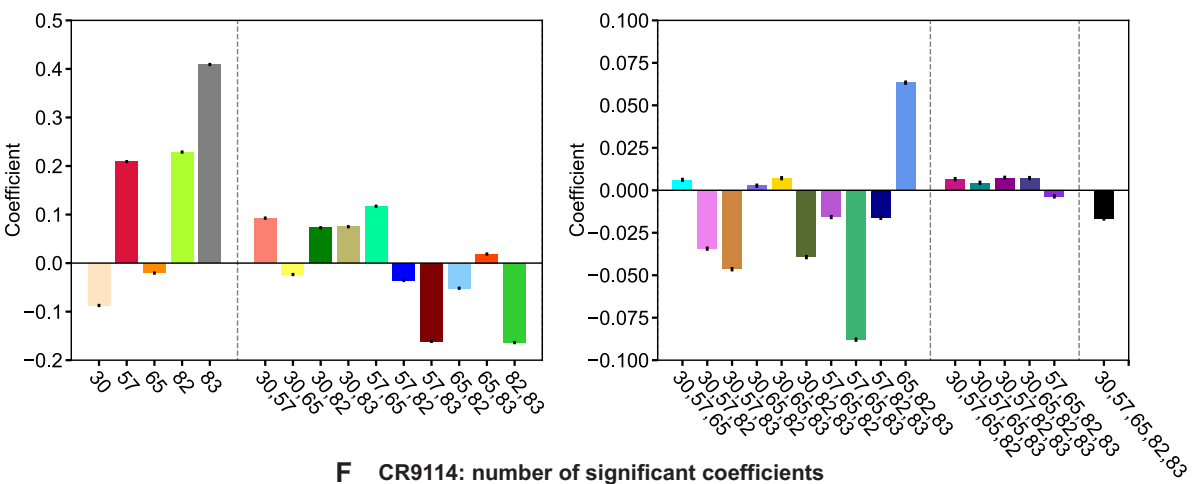

F CR9114: number of significant coefficients

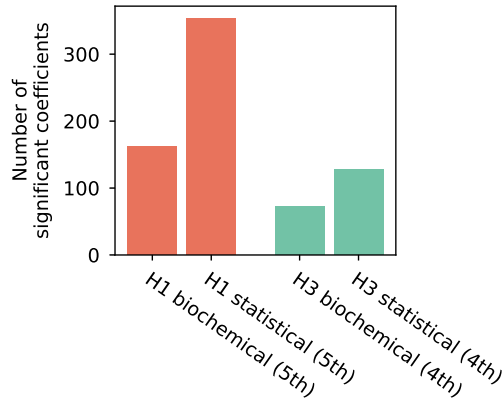

Appendix 2-figure 1. Results from statistical epistasis models for CR9114. (A) First-order effects, as in Figure 2A. ' $\mathrm{R}$ ' indicates required mutations. (B) Second-order effects for $\mathrm{H} 1$ (top right) and $\mathrm{H} 3$ (lower left), as in Figure 2D. Interactions with required mutations for $\mathrm{H} 3$ are noted in dark red. (C) Cumulative higher-order effects for CR9114 binding to H1, as in Figure 3A. (D) Cumulative higherorder effects for CR9114 binding to H3, as in Figure 3-figure supplement 3. (E) Inferred interaction coefficients for the set of five key epistatic loci, as in Figure 3-figure supplement 1B with corresponding colors. Note the different $y$-axis scales for the two subplots. Different interaction orders are separated by dotted lines. (F) Number of significant coefficients at all orders for the biochemical and statistical epistasis models. The maximal order of interaction for each model is indicated in parentheses. 

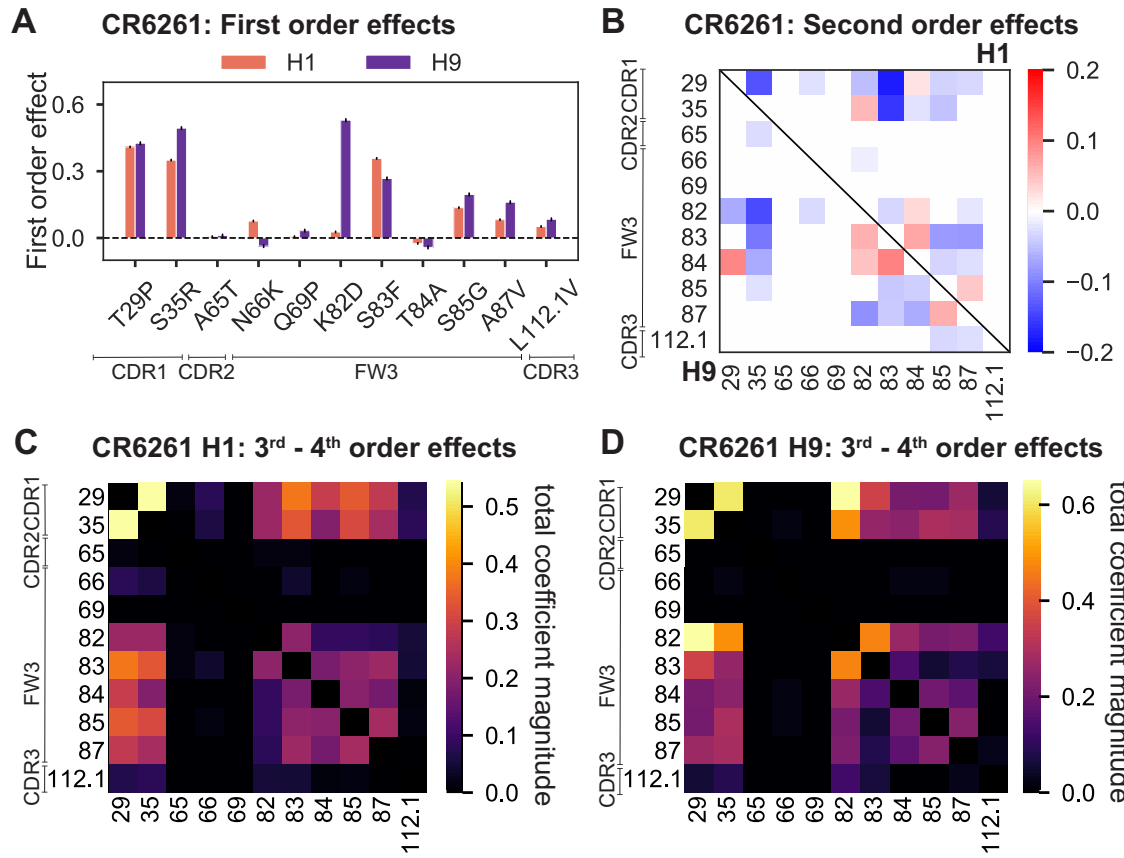

D CR6261 H9: $3^{\text {rd }}-4^{\text {th }}$ order effects

E CR6261: number of significant coefficients

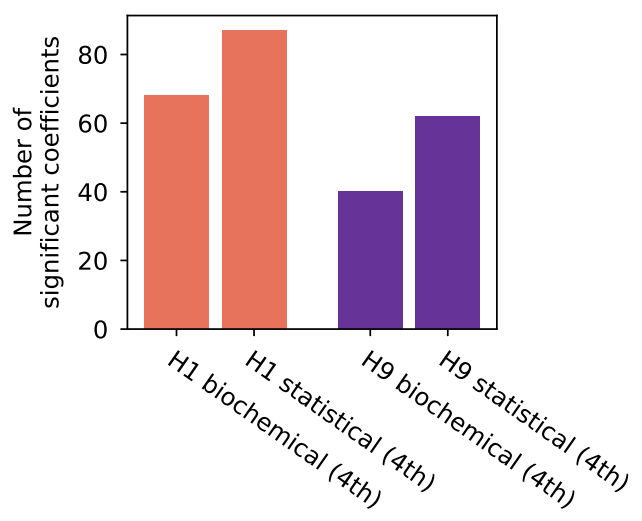

Appendix 2-figure 2. Results from statistical epistasis models for CR6261. (A) First-order effects, as in Figure 2B. (B) Second-order effects for H1 (top right) and H9 (lower left), as in Figure 2E. (C) Cumulative higher-order effects for CR6261 binding to H1, as in Figure 4A. (D) Cumulative higherorder effects for CR9114 binding to H9, as in Figure 4-figure supplement 2A. (E) Number of significant coefficients at all orders for the biochemical and statistical epistasis models. The maximal order of interaction for each model is indicated in parentheses. 
A

CR9114 statistical epistasis variance partitioning
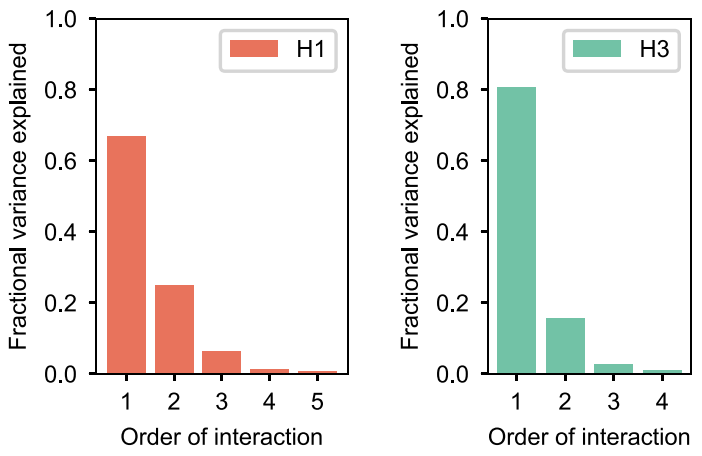

B CR6261 statistical epistasis variance partitioning

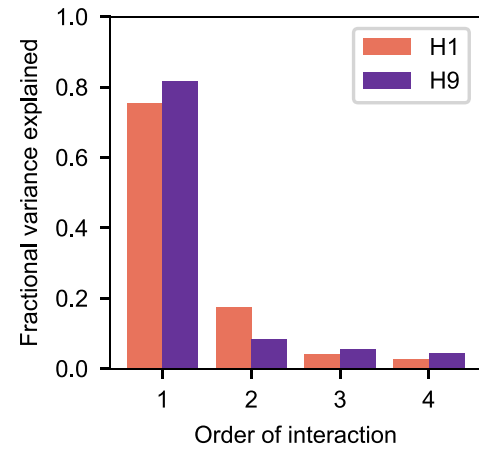

Appendix 2-figure 3. Variance partitioning of statistical epistasis models. (A) Variance partitioning for CR9114 binding to H1 (left) and H3 (right). (B) Variance partitioning for CR9114 binding to H1 and $\mathrm{H}$, denoted by colors as indicated.

In particular, another alternative approach to the inference of epistasis is to infer a full $L^{\text {th }}$-order model rather than truncating to lower order. This approach calculates $2^{L}$ epistatic coefficients, one for every datapoint, which allows for the detection of strong interactions at any order with the caveat that many coefficients may simply reflect experimental noise, especially for higher-order terms. We explore this approach by following Poelwijk et alo, 2019: we calculate epistatic coefficients using a Walsh-Hadamard transform of the $-\log _{10} K_{D}$ values, and calculate standard errors on each coefficient via error propagation using the standard errors of the data. We define significant coefficients by a $p$-value cutoff of 0.05 , with Bonferroni correction by the total number of parameters in the model (here $2^{L}$ ). We find that for all antibody-antigen combinations, this approach finds more significant coefficients than the optimal truncated models, many of which are at higher interaction orders than allowed in the truncated model (Appendix 2-figure 4). This analysis requires a measurement of $-\log _{10} K_{D}$ for every single variant, so we use data that has not been filtered for goodness-of-fit or error in the inference of binding affinity (see Methods), including some sequences that have substantial error. Therefore we prefer to use the more conservative regression approach for our in-depth analysis of epistasis; this inference at full order confirms the existence, strength, and identity of the high-order interactions we discuss from the regression approach, while also indicating that additional and even higher-order terms may yet exist. 
A CR9114: number of significant coefficients

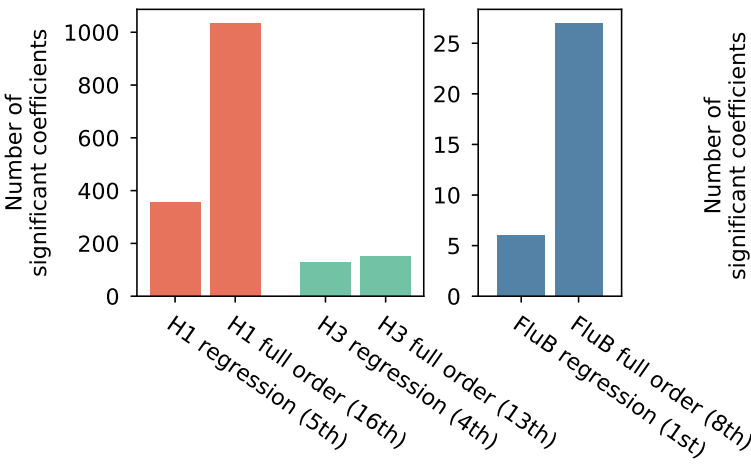

C CR9114: distribution of significant coefficients

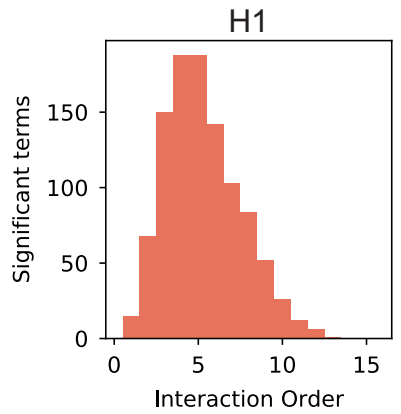

$\mathrm{H} 3$

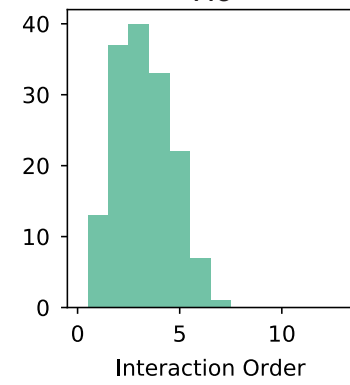

B CR6261: number of significant coefficients

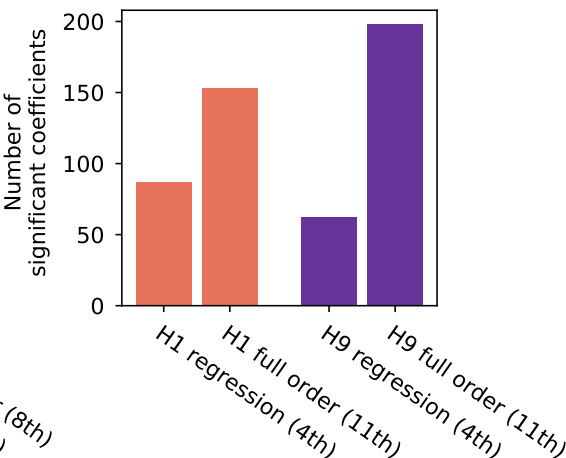

D CR6261: distribution of significant coefficients
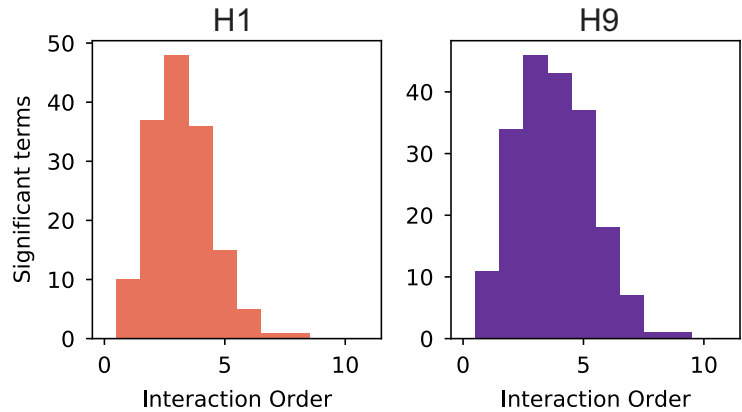

Appendix 2-figure 4. Epistasis inference at full order. (A,B) Numbers of significant coefficients for the full-order inference compared to optimal truncated regression models for (A) CR9114 and (B) CR6261. Significance for both model types is determined by $p<0.05$ with Bonferroni correction by the number of model parameters. (C,D) Distribution of interaction orders of significant coefficients for (C) CR9114 and (D) CR6261.

\section{Nonlinear models}

An alternative approach to understanding epistasis is to view nonlinearities in observed phenotype data as arising from a simple nonlinear transformation applied to an underlying, unobserved additive phenotype. In this view, a simple nonlinear "global epistasis" function with few parameters may describe the landscape as well or better than models of the sort described above, with their large number of "idiosyncratic epistasis" parameters. Many studies in other proteins have attempted to disentangle such global epistasis from idiosyncratic effects (Sailer and Harms, 2017a; Domingo et al., 2019; Sarkisyan et alı, 2016; Otwinowski et al., 2018; Otwinowski, 2018; Adams et al., 2019).

We already implement one global nonlinear transformation, by log-transforming our binding affinity measurements so that they are proportional to free energy changes, as described above. However, it is possible that another nonlinear transformation would capture the effects of many specific interaction coefficients, if there is a single underlying additive scale. In this section, we explore 
this possibility following the approach taken by Sailer and Harms, 2017a: we infer a nonlinear transformation that fits the phenotype data, invert it to "linearize" the phenotypes, re-fit interaction models on the linearized phenotypes, and then compare those model coefficients to the original coefficients to evaluate the role of the nonlinear transformation.

Our new model is

$$
y_{s}=\Phi\left(y_{s, \text { add }} ; k_{m}\right)=\Phi\left(\beta_{0}+\sum_{i}^{L} \beta_{i} x_{i, s} ; k_{m}\right),
$$

where $y_{s}$ are the observed phenotypes ( $-\log _{10} K_{D}$ values), $\Phi$ is a nonlinear function with a small number of associated parameters $k_{m}$, and $y_{s, \text { add }}$ are the underlying additive-scale phenotypes, parametrized as before by additive coefficients $\beta_{i}$.

To specify $\Phi$, we must choose a family of nonlinear functions. Typical choices include splines (Otwinowski et al., 2018) or power transforms (Sailer and Harms, 2017b). We found that logistic (sigmoid) functions fit our data better than power transforms or splines, and they are monotonic and invertible. Specifically, our logistic function with four parameters is

$$
\Phi(y ; A, B, \mu, \sigma)=\frac{A}{1+e^{\frac{(y-\mu)}{\sigma}}}+B .
$$

Logistic functions capture two features that we observe: first, there is a saturation effect at low values of $-\log _{10} K_{D}$, corresponding to nonspecific binding that our measurements are unable to distinguish (Batista and Neuberger, 1998); and second, for most antibody-antigen combinations we observe a saturation effect at moderately high values of $-\log _{10} K_{D}$. This latter effect is not due to limits on our measurement capabilities, as illustrated by higher values of $-\log _{10} K_{D}$ measured for the CR6261 library to $\mathrm{H} 9$ compared to values of $-\log _{10} K_{D}$ measured for the CR9114 library to H1, but instead due to widespread "diminishing returns" epistasis.

After specifying the functional form of $\Phi$, we must fit both the nonlinear parameters $k_{m}$ and underlying linear parameters $\beta_{i}$. In principle, one could fit all parameters jointly, using for example a maximum likelihood approach (Otwinowski et al., 2018). However, we take the simpler approach as implemented in the software package from Sailer and Harms, 2017a, which first infers the additive parameters $\beta_{i}$ from the observed phenotypes and then infers the nonlinear function parameters $k_{m}$. We show the resulting fit of $\Phi$ in Appendix 2-figure 5a for two representative examples, by plotting our estimate of the additive phenotypes $y_{s, \text { add }}$ on the $x$-axis and our observed phenotypes from data on the $y$-axis. We found that this simple procedure identified well-fitting $\Phi$ in a single step, and successive iterations did not significantly improve the fit.

After fitting the nonlinear transformation, we apply the inverse transformation to our observed phenotypes to obtain "linearized" phenotypes $y_{s, \text { lin }}$ :

$$
y_{s, \text { lin }}=\Phi^{-1}\left(y_{s}, k_{m}\right) .
$$

Because the fit of $\Phi$ is not perfect, the linearized phenotypes $y_{s, \text { lin }}$ are not exactly equal to the estimated additive phenotypes $y_{s, \text { add }}$, although linear regression on both quantities produces extremely similar values of $\beta_{i}$. For values that lie above the domain of $\Phi^{-1}$, we pin them to the largest estimated additive phenotype.

Finally, we can take our linearized phenotypes $y_{s, \text { lin }}$ and infer interaction model coefficients $\beta^{\prime}$ of various orders, exactly as described above for the untransformed "raw" phenotypes:

$$
y_{s, \operatorname{lin}}=\beta_{0}^{\prime}+\sum_{i} \beta_{i}^{\prime} x_{i, s}+\sum_{i<j}^{L} \beta_{i j}^{\prime} x_{i, s} x_{j, s}+\sum_{i<j<k}^{L} \beta_{i j k}^{\prime} x_{i, s} x_{j, s} x_{k, s}+\ldots+\varepsilon .
$$

We again perform this analysis in both the biochemical and statistical epistasis frameworks. If the inverse transformation has removed most or all of the nonlinearity, then the resulting optimal interaction models should be smaller (lower maximum order of interaction and/or fewer significant interaction coefficients).

Instead, we find that in all cases, the optimal order of interaction is unchanged or only decreased by one when inferring on linearized vs raw phenotypes. Specifically, the new (vs old) optimal orders 
are: 4th (vs 5th) for CR9114 binding to H1, 4th (vs 4th) for CR9114 binding to H3, 3rd (vs 4th) for CR6261 binding to H1, 3rd (vs 4th) for CR6261 binding to H9 in the biochemical epistasis framework, and 4th (vs 4th) for CR6261 binding to H9 in the statistical epistasis framework. We can compare the numbers of significant coefficients in these optimal models inferred on linearized phenotypes to the models with the same maximum order inferred on raw phenotypes (Appendix 2-figure 5d,e), where we see that the numbers are relatively comparable.

We next examine changes in the individual coefficients between these models. In Appendix 2 figure $5 \mathrm{~b}$, we show two representative scatterplots between the raw phenotype coefficients $\beta$ and the linearized phenotype coefficients $\beta^{\prime}$, where only significant coefficients are shown for clarity. While some coefficients show dramatic changes, overall the two sets of coefficients are quite well correlated. To see which sites are involved in strong changes, we can also represent coefficient changes in a heatmap format (Appendix 2-figure 5c). Here, diagonal cells show the change in coefficient for single sites $\left(\beta_{i}^{\prime}-\beta_{i}\right)$, while off-diagonal cells show the sum of coefficient changes over all pairwise and higher terms involving each pair of mutations. We observe that for some antibodyantigen pairs, such as CR9114 binding to H1, the strongest net changes are negative, though not negative enough to remove the many significant coefficients. For other antibody-antigen pairs such as CR6261 binding to $\mathrm{H} 1$, there are both positive and negative net changes, indicating that the nonlinear transformation is changing the epistatic landscape rather than correcting for it.

A Fitted logistic functions

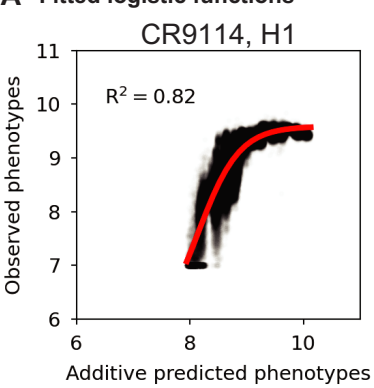

CR6261, H1

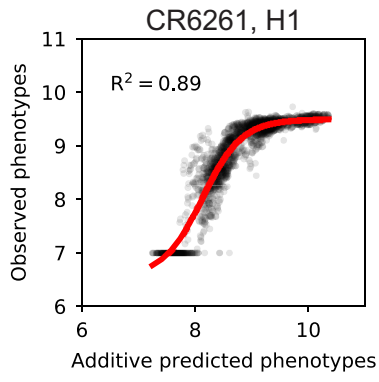

D CR9114 significant coefficients

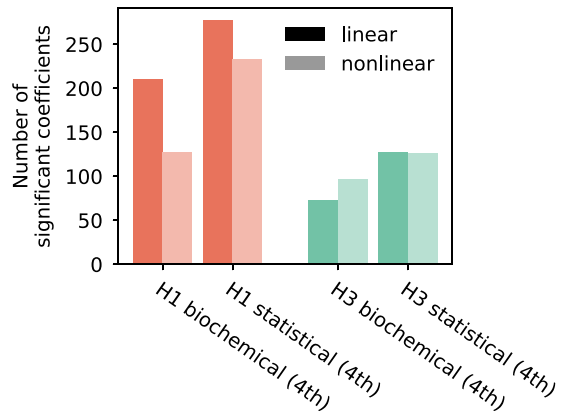

B Coefficient correlation for models at optimal order
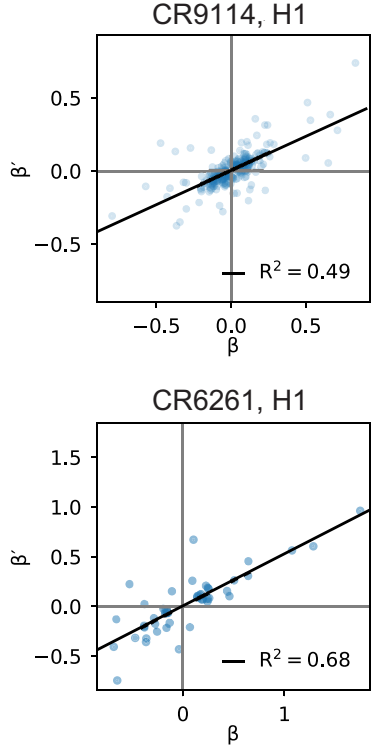

C Coefficient net changes for models at optimal order
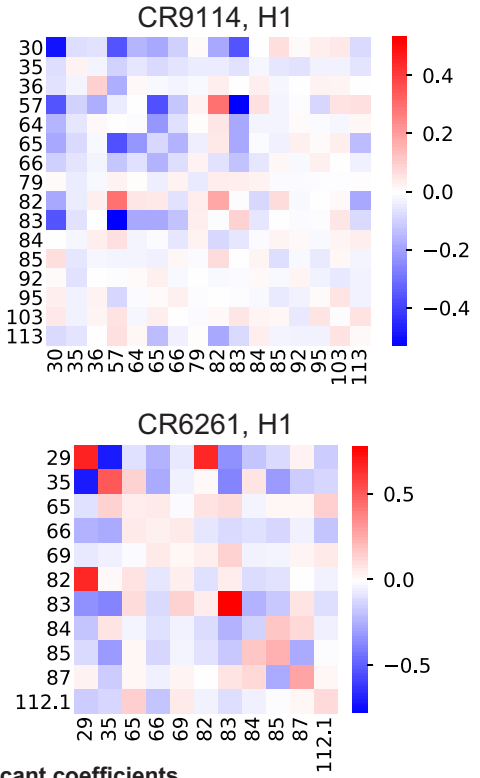

E CR6261 significant coefficients

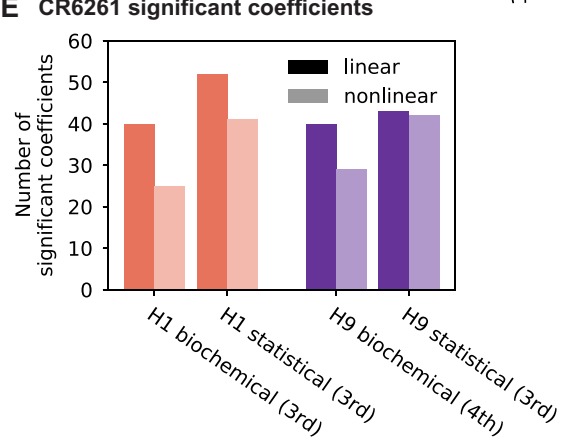

Appendix 2-figure 5. Results from epistasis models with nonlinear transformations. (A), Fitting logistic functions to additive predicted phenotypes. Red lines indicate the optimized logistic function $\Phi$, with $R^{2}$ as indicated. (B), Scatterplot of coefficients $\beta^{\prime}$ from the optimal order model inferred on linearized data (after inverting the best-fit nonlinear transformation) against original Appendix 2-figure 5 continued on next page 
Appendix 2-figure 5 continued

coefficients $\beta$ for the model with the same maximum order. (C), Net changes of coefficients by site. Diagonal cells show changes in linear coefficients. Off-diagonal cells show the sum of changes over terms at all orders (2nd and above) in which the given pair of mutations is involved. For (A-C), we show two representative antibody-antigen combinations: CR9114 binding to H1, top, and CR6261 binding to $H 1$, bottom. $(D, E)$, Number of significant coefficients in optimal order models fit to phenotypes transformed by the inverse nonlinear function (light bars), compared to original coefficients from linear models with the same maximal order (dark bars), for (D) CR9114 and (E) CR6261. The epistasis type and model order are indicated on the $\mathrm{x}$-axis.

In summary, we find that nonlinear logistic transformations can account for a portion of the nonlinearities observed in our data, sometimes reducing the maximal order of interaction by one. However, all antigen-antibody pairs still exhibit strong idiosyncratic epistasis up to at least third order after correcting for global epistasis, and the resulting numbers and magnitudes of significant coefficients are not drastically changed. Thus, it does not appear that global epistasis can explain our data much more simply than models with individual interactions, and so we confine our main analysis to idiosyncratic epistasis models. 